\title{
Second-Order Systems of ODEs Admitting Three-Dimensional Lie Algebras and Integrability
}

\author{
Muhammad Ayub, ${ }^{1,2}$ Masood Khan, ${ }^{1}$ and F. M. Mahomed ${ }^{2}$ \\ ${ }^{1}$ Department of Mathematics, Quaid-i-Azam University, Islamabad 45320, Pakistan \\ ${ }^{2}$ Centre for Differential Equations, Continuum Mechanics and Applications, School of Computational and Applied Mathematics, \\ University of the Witwatersrand, Wits 2050, South Africa
}

Correspondence should be addressed to F. M. Mahomed; fazal.mahomed@wits.ac.za

Received 27 November 2012; Accepted 20 January 2013

Academic Editor: Mehmet Pakdemirli

Copyright (C) 2013 Muhammad Ayub et al. This is an open access article distributed under the Creative Commons Attribution License, which permits unrestricted use, distribution, and reproduction in any medium, provided the original work is properly cited.

We present a systematic procedure for the determination of a complete set of $k$ th-order $(k \geq 2)$ differential invariants corresponding to vector fields in three variables for three-dimensional Lie algebras. In addition, we give a procedure for the construction of a system of two $k$ th-order ODEs admitting three-dimensional Lie algebras from the associated complete set of invariants and show that there are 29 classes for the case of $k=2$ and 31 classes for the case of $k \geq 3$. We discuss the singular invariant representations of canonical forms for systems of two second-order ODEs admitting three-dimensional Lie algebras. Furthermore, we give an integration procedure for canonical forms for systems of two second-order ODEs admitting three-dimensional Lie algebras which comprises of two approaches, namely, division into four types I, II, III, and IV and that of integrability of the invariant representations. We prove that if a system of two second-order ODEs has a three-dimensional solvable Lie algebra, then, its general solution can be obtained from a partially linear, partially coupled or reduced invariantly represented system of equations. A natural extension of this result is provided for a system of two $k$ th-order $(k \geq 3)$ ODEs. We present illustrative examples of familiar integrable physical systems which admit three-dimensional Lie algebras such as the classical Kepler problem and the generalized Ermakov systems that give rise to closed trajectories.

\section{Introduction}

Realizations of Lie algebras in terms of vector fields are applied, in particular, for the integration and classification of ordinary differential equations (see, e.g. [1-5]). In spite of the importance for applications, the problem of complete description of realizations has not been solved even for cases when either the dimension of the algebras or the dimension of the realization space is a fixed small integer [6]. The classification of finite-dimensional Lie algebras was initiated by Lie [7] who also presented realizations in terms of vector fields in $(1+1)$ - and $(1+2)$-dimensional spaces. In the case of vector fields in the two-dimensional space, he gave a complete classification in the complex domain. For realizations of vectors fields in the three-dimensional space, Lie presented a partial classification which was recently completed. All the possible complex Lie algebras of dimension less than four were already obtained by Lie himself $[8,9]$. Bianchi [10] investigated three-dimensional real Lie algebras. Complete and correct classification of four-dimensional real Lie algebras was obtained by Mubarakzjanov [11]. Wafo Soh and Mahomed [12] used the results of Bianchi and Mubarakzjanov to classify realizations of three- and fourdimensional real Lie algebras in the space of three variables, and they also described systems of two second-order ODEs admitting real four-dimensional Lie algebras.

The realizations of real Lie algebras of dimension no greater than four of vector fields in the space of an arbitrary (finite) number of variables were given in [6]. Popovych et al. [6] used the results of Bianchi and Mubarakzjanov with a different approach. They found that the work [12] does not provide the complete list of realizations of real Lie algebras of dimensions three and four. 
Systems of two second-order ODEs arise frequently in several applications in classical, fluid, and quantum mechanics as well as in general relativity. Hence, these have been studied vigorously over the years. A sample of the literature as far as symmetry Lie algebras of ODEs are concerned can be found in [13-15]. In particular, the classification of systems of two second-order ODEs admitting real four-dimensional Lie algebras was attempted in [12]. Gaponova and Nesterenko [16] gave a classification of system of two second-order ODEs admitting real three- and four-dimensional Lie algebras by using the results of [6].

In the this paper, by utilizing realizations given in [6], a systematic procedure for finding a complete set of $k$ thorder $(k \geq 2)$ differential invariants including basis of invariants corresponding to vector fields in three variables for three-dimensional Lie algebras is presented. In addition, we give a procedure for the construction of two $k$ th-order ODEs possessing three-dimensional Lie algebras from the associated complete set of invariants. We show that there are 29 classes for $k=2$ and 31 classes for $k \geq 3$. Moreover, we mention those cases in which regular systems of two second-order ODEs are not obtained for threedimensional Lie algebras. We present a brief discussion on the singular representation of canonical forms for systems of two second-order ODEs admitting three-dimensional Lie algebras. Furthermore, we provide an integration procedure for canonical forms for systems of two second-order ODEs admitting three-dimensional Lie algebras. This procedure is composed of two approaches, namely, one which depends on general observations of canonical forms which admit threedimensional Lie algebras; the second depends on a complete set of differential invariants and is applicable only for those cases which admit three-dimensional solvable Lie algebras. We show by familiar physical examples how these integration approaches work. In the second approach, for solvable Lie algebras, we analyze integrability in terms of integration of the invariant representations. We prove that if a system of two second-order ODEs admits a solvable three-dimensional Lie algebra, then, its general solution can be deduced from a partially linear, partially coupled or invariant reduced system of equations. A natural extension of this result is stated for a system of two $k$ th-order $(k \geq 3)$ ODEs.

In Table 1, for ease of reference, we list the realizations of three-dimensional Lie algebras as given in [6]. In the second section, for the Lie algebras of vector fields considered, we discuss and deduce a complete set of differential invariants including basis of invariants with the construction of the corresponding systems of two $k$ th-order ODEs. Moreover, for systems of two second-order ODEs admitting threedimensional Lie algebras, we discuss the singular representation and those cases in which we do not obtain systems. In the end, in Table 2, we give a complete list of secondorder differential invariants corresponding to vector fields in three variables of three-dimensional real Lie algebras and the associated complete list of canonical forms for systems of two second-order ODEs. In Section 3, we provide an integration procedure for the canonical forms of systems of two $k$ th-order $(k \geq 2)$ ODEs admitting three-dimensional Lie algebras. This is stated as theorems for systems admitting solvable algebras.
Reduction of canonical forms for systems of two secondorder ODEs into invariant systems of first-order and algebraic equations are given in Table 2. The paper ends with a brief conclusion.

\section{Invariants and Systems of ODEs}

When one calculates the symmetries of a given differential equation, one finds the generators in the form of vector fields and then computes the Lie brackets to get the structure constants of the particular Lie algebra one has found. We can start from a given Lie algebra with a set of structure constants and ask which vector fields in at most three variables satisfy the given Lie bracket relations with none of the vector fields vanishing. We thus ask for possible realizations of the given Lie algebra. We make use of the realizations of three-dimensional Lie algebras in the (1+2)-dimensional space given in [6]. We utilize these realizations in order to obtain differential invariants and invariant representations for system of two second-order and higher-order ODEs. Extensions to higher order invariants are given to understand the behavior of such invariants. This is the inverse problem in symmetry analysis and was initiated by Lie.

For completeness and ease of reference, we list the realizations of three-dimensional real algebras as given in [6] in Table 1. However, we mostly keep the format of [12].

Differential invariants play an important role in the structure and reduction of differential equations. Differential invariants can be obtained by various approaches and we refer, for example, to [16] for realizations in $(1+2)$ dimensional space. Differential invariants obtained by various approaches corresponding to three-dimensional Lie algebras have very interesting properties. In the investigation of the invariant approach for a system of two second-order ODEs admitting three-dimensional Lie algebras, it is found that, for a regular system of ODEs, there are four independent invariants, three of which play the role of basis and the fourth one is derived from the remaining three.

For the case of a regular system of two second-order ODEs admitting three-dimensional Lie algebras, there are four functionally independent invariants. For example, consider the system of two second-order ODEs

$$
\frac{\ddot{x}}{\dot{x}^{2}}=f\left(y, \frac{\dot{x}}{\dot{y}}\right), \quad \frac{\ddot{x}}{\dot{y}^{2}}-\frac{\dot{x} \ddot{y}}{\dot{y}^{3}}=g\left(y, \frac{\dot{x}}{\dot{y}}\right),
$$

admitting the three-dimensional Lie algebra $A_{3,2}^{3}$. We have the four independent invariants

$$
w_{1}=y, \quad w_{2}=\frac{\dot{x}}{\dot{y}}, \quad w_{3}=\frac{\ddot{x}}{\dot{x}^{2}}, \quad w_{4}=\frac{\ddot{x}}{\dot{y}^{2}}-\frac{\dot{x} \ddot{y}}{\dot{y}^{3}} .
$$

Here, the invariants $w_{3}$ and $w_{4}$ are of order two, that is, equal to the order of the system of ODEs that is constructible. 
TABLE 1: Realizations of three-dimensional Lie algebras in three variables.

\begin{tabular}{|c|c|c|c|}
\hline Algebra & $N$ & Realization & Rank \\
\hline \multirow{4}{*}{$A_{3,1}$} & 1 & $\partial_{t}, \partial_{x}, \partial_{y}$ & 3 \\
\hline & 2 & $\partial_{x}, \partial_{y}, t \partial_{x}+f(t) \partial_{y}$ & 2 \\
\hline & 3 & $\partial_{x}, t \partial_{x}, y \partial_{x}$ & 1 \\
\hline & 4 & $\partial_{t}, x \partial_{t}, \phi(x) \partial_{t}, \quad \phi^{\prime \prime}(x) \neq 0$ & 1 \\
\hline \multirow{4}{*}{$\begin{array}{l}A_{3,2} \\
{\left[X_{1}, X_{2}\right]=X_{1}}\end{array}$} & 1 & $\partial_{t}, t \partial_{t}+\partial_{y}, \partial_{x}$ & 3 \\
\hline & 2 & $\partial_{t}, t \partial_{t}+y \partial_{x}, \partial_{x}$ & 2 \\
\hline & 3 & $\partial_{t}, t \partial_{t}, \partial_{x}$ & 2 \\
\hline & 4 & $\partial_{x}, t \partial_{t}+x \partial_{x}, t \partial_{x}$ & 2 \\
\hline \multirow{3}{*}{$\begin{array}{l}A_{3,3} \\
{\left[X_{2}, X_{3}\right]=X_{1}}\end{array}$} & 1 & $\partial_{t}, \partial_{x}, x \partial_{t}$ & 2 \\
\hline & 2 & $\partial_{t}, \partial_{x}, x \partial_{t}+y \partial_{x}$ & 2 \\
\hline & 3 & $\partial_{t}, \partial_{x}, x \partial_{t}+\partial_{y}$ & 3 \\
\hline$A_{3,4}$ & 1 & $\partial_{t}, \partial_{x},(t+x) \partial_{t}+x \partial_{x}$ & 2 \\
\hline$\left[X_{1}, X_{3}\right]=X_{1}$ & 2 & $\partial_{t}, \partial_{x},(t+x) \partial_{t}+x \partial_{x}+\partial_{y}$ & 3 \\
\hline$\left[X_{2}, X_{3}\right]=X_{1}+X_{2}$ & 3 & $\partial_{x}, t \partial_{x},-\partial_{t}+x \partial_{x}$ & 2 \\
\hline \multirow{4}{*}{$\begin{array}{l}A_{3,5} \\
{\left[X_{1}, X_{3}\right]=X_{1}} \\
{\left[X_{2}, X_{3}\right]=X_{2}}\end{array}$} & 1 & $\partial_{t}, \partial_{x}, t \partial_{t}+x \partial_{x}$ & 2 \\
\hline & 2 & $\partial_{t}, \partial_{x}, t \partial_{t}+x \partial_{x}+\partial_{y}$ & 3 \\
\hline & 3 & $\partial_{x}, t \partial_{x}, x \partial_{x}$ & 1 \\
\hline & 4 & $\partial_{x}, t \partial_{x}, x \partial_{x}+\partial_{y}$ & 2 \\
\hline$A_{3,6}^{a},|a| \leq 1, a \neq 0,1$ & 1 & $\partial_{t}, \partial_{x}, t \partial_{t}+a x \partial_{x}$ & 2 \\
\hline$\left[X_{1}, X_{3}\right]=X_{1}$ & 2 & $\partial_{t}, \partial_{x}, t \partial_{t}+a x \partial_{x}+\partial_{y}$ & 3 \\
\hline$\left[X_{2}, X_{3}\right]=a X_{2}$ & 3 & $\partial_{x}, t \partial_{x},(1-a) t \partial_{t}+x \partial_{x}$ & 2 \\
\hline$A_{3,7}^{a}, a \geq 0$ & 1 & $\partial_{t}, \partial_{x},(a t+x) \partial_{t}+(a x-t) \partial_{x}$ & 2 \\
\hline$\left[X_{1}, X_{3}\right]=a X_{1}-X_{2}$ & 2 & $\partial_{t}, \partial_{x},(a t+x) \partial_{t}+(a x-t) \partial_{x}+\partial_{y}$ & 3 \\
\hline$\left[X_{2}, X_{3}\right]=X_{1}+a X_{2}$ & 3 & $\partial_{x}, t \partial_{x},-\left(1+t^{2}\right) \partial_{t}+(a-t) x \partial_{x}$ & 2 \\
\hline \multirow{5}{*}{$\begin{array}{l}A_{3,8},\{\operatorname{sl}(2, R)\} \\
{\left[X_{1}, X_{2}\right]=X_{1}} \\
{\left[X_{2}, X_{3}\right]=X_{3}} \\
{\left[X_{3}, X_{1}\right]=-2 X_{2}}\end{array}$} & 1 & $\partial_{t}, t \partial_{t}, t^{2} \partial_{t}$ & 1 \\
\hline & 2 & $\partial_{t}, t \partial_{t}+x \partial_{x}, t^{2} \partial_{t}+2 t x \partial_{x}+x \partial_{y}$ & 3 \\
\hline & 3 & $\partial_{t}+\partial_{x}, t \partial_{t}+x \partial_{x}, t^{2} \partial_{t}+x^{2} \partial_{x}$ & 2 \\
\hline & 4 & $-t \partial_{x}, \frac{1}{2}\left(-t \partial_{t}+x \partial_{x}\right), x \partial_{t}$ & 2 \\
\hline & 5 & $\partial_{t}, t \partial_{t}+x \partial_{x},\left(t^{2}-x^{2}\right) \partial_{t}+2 t x \partial_{x}$ & 2 \\
\hline $\begin{array}{l}A_{3,9},\{\operatorname{so}(3)\} \\
{\left[X_{1}, X_{2}\right]=X_{3}}\end{array}$ & 1 & $\left(1+t^{2}\right) \partial_{t}+x t \partial_{x}, x \partial_{t}-t \partial_{x},-x t \partial_{t}-\left(1+x^{2}\right) \partial_{x}$ & 2 \\
\hline $\begin{array}{l}{\left[X_{3}, X_{1}\right]=X_{2}} \\
{\left[X_{2}, X_{3}\right]=X_{1}}\end{array}$ & 2 & $-\sin t \tan x \partial_{t}-\cos t \partial_{x}+\sin t \sec x \partial_{y},-\cos t \tan x \partial_{t}+\sin t \partial_{x}+\cos t \sec x \partial_{y}, \partial_{t}$ & 3 \\
\hline
\end{tabular}

Similarly for the regular system of two third-order ODEs

$$
\begin{gathered}
\frac{\ddot{x}}{\dot{x}^{2} \dot{y}}-\frac{2 \ddot{x}^{2}}{\dot{x}^{3} \dot{y}}=f\left(y, \frac{\dot{x}}{\dot{y}}, \frac{\ddot{x}}{\dot{x}^{2}}, \frac{\ddot{x}}{\dot{y}^{2}}-\frac{\dot{x} \ddot{y}}{\dot{y}^{3}}\right), \\
-\frac{\dot{x} \ddot{y}}{\dot{y}^{4}}+\frac{\ddot{x} \dot{y}^{2}-3 \ddot{x} \dot{y} \ddot{y}+3 \dot{x} \ddot{y}^{2}}{\dot{y}^{5}}=g\left(y, \frac{\dot{x}}{\dot{y}}, \frac{\ddot{x}}{\dot{x}^{2}}, \frac{\ddot{x}}{\dot{y}^{2}}-\frac{\dot{x} \ddot{y}}{\dot{y}^{3}}\right),
\end{gathered}
$$

admitting the same Lie algebra $A_{3,2}^{3}$; we have the six invariants

$$
\begin{aligned}
& w_{1}=y, \quad w_{2}=\frac{\dot{x}}{\dot{y}}, \quad w_{3}=\frac{\ddot{x}}{\dot{x}^{2}}, \quad w_{4}=\frac{\ddot{x}}{\dot{y}^{2}}-\frac{\dot{x} \ddot{y}}{\dot{y}^{3}}, \\
& w_{5}=\frac{\ddot{x}}{\dot{x}^{2} \dot{y}}-\frac{2 \ddot{x}^{2}}{\dot{x}^{3} \dot{y}}, \quad w_{6}=-\frac{\dot{x} \ddot{y}}{\dot{y}^{4}}+\frac{\dddot{x} \dot{y}^{2}-3 \ddot{x} \dot{y} \ddot{y}+3 \dot{x} \ddot{y}^{2}}{\dot{y}^{5}},
\end{aligned}
$$

from which we determine the third-order system. Here, the invariants $w_{5}$ and $w_{6}$ are of order three, that is, equal to the order of the considered system of ODEs. By proceeding in this way for regular systems of two $k$ th-order ODEs admitting three-dimensional Lie algebras, the number of order $k$ invariants is two.

To study differential invariants further, we consider the following example. Consider the system

$$
\begin{gathered}
\frac{\ddot{x}}{\dot{x} \dot{y}}=f\left(e^{y} \dot{y}, \dot{x} \dot{y}^{a-1}\right), \\
\frac{e^{-y} \ddot{x} \dot{y}^{a-1}}{\dot{y}^{2}+\ddot{y}}+\frac{(a-1) e^{-y} \dot{x} \dot{y}^{a-2} \ddot{y}}{\dot{y}^{2}+\ddot{y}}=g\left(e^{y} \dot{y}, \dot{x} \dot{y}^{a-1}\right),
\end{gathered}
$$


TABLE 2: Second-order differential invariants and equations.

\begin{tabular}{|c|c|c|}
\hline Algebra & $N$ & Invariants and equations \\
\hline$A_{3,1}$ & 3 & $\begin{array}{l}u=\dot{x}, v=\dot{y}, w=\ddot{x}, \frac{d v}{d u}=\frac{\ddot{y}}{\ddot{x}} \\
\ddot{x}=f(u, v), \frac{\ddot{y}}{\ddot{x}}=g(u, v) \\
u=t, v=\dot{y}-\dot{x} f^{\prime}(t), w=\ddot{x}, \frac{d v}{d u}=\ddot{y}-\ddot{x} f^{\prime}(t)-\dot{x} f^{\prime \prime}(t) \\
\ddot{x}=h(u, v), \ddot{y}-\ddot{x} f^{\prime}(t)-\dot{x} f^{\prime \prime}(t)=k(u, v) \\
u=t, v=y, w=\ddot{x}, \frac{d v}{d u}=\dot{y}, \text { and } \ddot{y}=0 \\
\ddot{x}=f\left(u, v, \frac{d v}{d u}\right), \ddot{y}=0\end{array}$ \\
\hline$A_{3,2}$ & 4 & $\begin{array}{l}u=e^{y} \dot{y}, v=\frac{\dot{x}}{\dot{y}}, w=\frac{\ddot{x}}{\dot{x}^{2}}, \frac{d v}{d u}=\frac{\ddot{x}}{e^{y} \dot{y}\left(\dot{y}^{2}+\ddot{y}\right)}-\frac{\dot{x} \ddot{y}}{e^{y} \dot{y}^{2}\left(\dot{y}^{2}+\ddot{y}\right)} \\
\frac{\ddot{x}}{\dot{x}^{2}}=f(u, v), \frac{\dot{x}}{e^{y} \dot{y}\left(\dot{y}^{2}+\ddot{y}\right)}-\frac{\ddot{y}}{e^{y} \dot{y}^{2}\left(\dot{y}^{2}+\ddot{y}\right)}=g(u, v) \\
u=y, v=e^{\dot{x} / \dot{y}} \dot{y}, \quad w=\frac{\ddot{y}}{\dot{y}^{2}}, \frac{d v}{d u}=e^{\dot{x} / \dot{y}} \frac{\dot{x}}{\dot{y}}+e^{\dot{x} / \dot{y}} \frac{(\dot{y}-\dot{x}) \ddot{y}}{\dot{y}^{2}} \\
\frac{\ddot{y}}{\dot{y}^{2}}=f(u, v), e^{\dot{x} / \dot{y}} \frac{\ddot{x}}{\dot{y}}+e^{\dot{x} / \dot{y}} \frac{(\dot{y}-\dot{x}) \ddot{y}}{\dot{y}^{2}}=g(u, v) \\
u=y, v=\frac{\dot{x}}{\dot{y}}, w=\frac{\ddot{x}}{\dot{x}^{2}}, \frac{d v}{d u}=\frac{\ddot{x}}{\dot{y}^{2}}-\frac{\dot{x} \ddot{y}}{\dot{y}^{3}} \\
\frac{\ddot{x}}{\dot{x}^{2}}=f(u, v), \quad \frac{\ddot{x}}{\dot{y}^{2}}-\frac{\dot{x} \ddot{y}}{\dot{y}^{3}}=g(u, v) \\
u=y, v=t \dot{y}, w=t \ddot{x}, \frac{d v}{d u}=1+\frac{t \ddot{y}}{\dot{y}} \\
t \ddot{x}=f(u, v), 1+\frac{t \ddot{y}}{\dot{y}}=g(u, v)\end{array}$ \\
\hline$A_{3,3}$ & 3 & $\begin{array}{l}u=y, v=\frac{\dot{x}}{\dot{y}}, w=\frac{\ddot{x}}{\dot{x}^{3}}, \frac{d v}{d u}=\frac{\ddot{x}}{\dot{y}^{2}}-\frac{\dot{x} \ddot{y}}{\dot{y}^{3}} \\
\frac{\ddot{x}}{\dot{x}^{3}}=f(u, v), \frac{\ddot{x}}{\dot{y}^{2}}-\frac{\dot{x} \ddot{y}}{\dot{y}^{3}}=g(u, v) \\
u=y, \quad v=\frac{\dot{x}^{2}}{2 \dot{y}^{2}}-\frac{1}{\dot{y}}, \quad w=\frac{\dot{x} \ddot{y}}{\dot{y}^{3}}-\frac{\ddot{x}}{\dot{y}^{2}}, \frac{d v}{d u}=\frac{\dot{x} \ddot{x}}{\dot{y}^{3}}+\frac{\left(\dot{y}-\dot{x}^{2}\right) \ddot{y}}{\dot{y}^{4}} \\
\frac{\dot{x} \ddot{y}}{\dot{y}^{3}}-\frac{\ddot{x}}{\dot{y}^{2}}=f(u, v), \frac{\dot{x} \ddot{x}}{\dot{y}^{3}}+\frac{\left(\dot{y}-\dot{x}^{2}\right) \ddot{y}}{\dot{y}^{4}}=g(u, v) \\
u=y-\frac{1}{\dot{x}}, v=\frac{\dot{x}}{\dot{y}}, w=\frac{\ddot{x}}{\dot{x}^{3}}, \frac{d v}{d u}=\frac{\dot{x}^{2} \ddot{x}}{\dot{y}\left(\ddot{x}+\dot{y} \dot{x}^{2}\right)}-\frac{\dot{x}^{3} \ddot{y}}{\dot{y}^{2}\left(\ddot{x}+\dot{y} \dot{x}^{2}\right)} \\
\frac{\ddot{x}}{\dot{x}^{3}}=f(u, v), \frac{\dot{x}^{2} \ddot{x}}{\dot{y}\left(\ddot{x}+\dot{y} \dot{x}^{2}\right)}-\frac{\dot{x}^{3} \ddot{y}}{\dot{y}^{2}\left(\ddot{x}+\dot{y} \dot{x}^{2}\right)}=g(u, v)\end{array}$ \\
\hline$A_{3,4}$ & 2 & $\begin{array}{l}u=y, v=e^{1 / \dot{x}} \frac{\dot{y}}{\dot{x}}, w=\frac{\ddot{x}}{\dot{x}^{2} \dot{y}}, \frac{d v}{d u}=e^{1 / \dot{x}} \frac{\ddot{y}}{\dot{x} \dot{y}}-e^{1 / \dot{x}} \frac{(1+\dot{x}) \ddot{x}}{\dot{x}^{3}} \\
\frac{\ddot{x}}{\dot{x}^{2} \dot{y}}=f(u, v), e^{1 / \dot{x}} \frac{\ddot{y}}{\dot{x} \dot{y}}-e^{1 / \dot{x}} \frac{(1+\dot{x}) \ddot{x}}{\dot{x}^{3}}=g(u, v) \\
u=y-\frac{1}{\dot{x}}, v=e^{1 / \dot{x}} \frac{\dot{y}}{\dot{x}}, w=\frac{\ddot{x}}{\dot{x}^{2} \dot{y}}, \frac{d v}{d u}=e^{1 / \dot{x}} \frac{\dot{x} \ddot{y}}{\ddot{x}+\dot{x}^{2} \dot{y}}-e^{1 / \dot{x}} \frac{(1+\dot{x}) \ddot{x} \dot{y}}{\dot{x}\left(\ddot{x}+\dot{x}^{2} \dot{y}\right)} \\
\frac{\ddot{x}}{\dot{x}^{2} \dot{y}}=f(u, v), e^{1 / \dot{x}} \frac{\dot{x} \ddot{y}}{\ddot{x}+\dot{x}^{2} \dot{y}}-e^{1 / \dot{x}} \frac{(1+\dot{x}) \ddot{x} \dot{y}}{\dot{x}\left(\ddot{x}+\dot{x}^{2} \dot{y}\right)}=g(u, v) \\
u=y, v=\dot{y}, w=e^{t} \ddot{x}, \frac{d v}{d u}=\frac{\ddot{y}}{\dot{y}} \\
e^{t} \ddot{x}=f(u, v), \frac{\ddot{y}}{\dot{y}}=g(u, v)\end{array}$ \\
\hline$A_{3,5}$ & 1 & $\begin{array}{l}u=y, v=\dot{x}, w=\frac{\ddot{y}}{\dot{y}^{2}}, \frac{d v}{d u}=\frac{\ddot{x}}{\dot{y}} \\
\frac{\ddot{y}}{\dot{y}^{2}}=f(u, v), \frac{\ddot{x}}{\dot{y}}=g(u, v)\end{array}$ \\
\hline
\end{tabular}


TABLe 2: Continued.

\begin{tabular}{|c|c|c|}
\hline Algebra & $N$ & Invariants and equations \\
\hline & 3 & $\begin{array}{l}u=\dot{x}, v=e^{y} \dot{y}, w=\frac{\ddot{x}}{\dot{y}}, \frac{d v}{d u}=e^{y} \frac{\dot{y}^{2}}{\ddot{x}}+e^{y} \frac{\ddot{y}}{\ddot{x}} \\
\frac{\ddot{x}}{\dot{y}}=f(u, v), e^{y} \frac{\dot{y}^{2}}{\ddot{x}}+e^{y} \frac{\ddot{y}}{\ddot{x}}=g(u, v) \\
u=t, v=y, \frac{d v}{d u}=\dot{y}, \frac{d^{2} v}{d u^{2}}=\ddot{y}, \text { and } \ddot{x}=0 \\
\ddot{x}=0, \ddot{y}=g\left(u, v, \frac{d v}{d u}\right) \\
u=t, v=\dot{y}, w=e^{-y} \ddot{x}, \frac{d v}{d u}=\ddot{y} \\
e^{-y} \ddot{x}=f(u, v), \ddot{y}=g(u, v)\end{array}$ \\
\hline $\begin{array}{l}A_{3,6}^{a} \\
|a| \leq 1 \\
a \neq 0,1\end{array}$ & 3 & $\begin{array}{l}u=y, v=\dot{x} \dot{y}^{a-1}, w=\frac{\ddot{x}}{\dot{x} \dot{y}}, \frac{d v}{d u}=\ddot{x} \dot{y}^{a-2}+(a-1) \dot{x} \dot{y}^{a-3} \ddot{y} \\
\frac{\ddot{x}}{\dot{x} \dot{y}}=f(u, v), \ddot{x} \dot{y}^{a-2}+(a-1) \dot{x} \dot{y}^{a-3} \ddot{y}=g(u, v) \\
u=e^{y} \dot{y}, \quad v=\dot{x} \dot{y}^{a-1}, w=\frac{\ddot{x}}{\dot{x} \dot{y}}, \frac{d v}{d u}=\frac{e^{-y} \ddot{x} \dot{y}^{a-1}}{\dot{y}^{2}+\ddot{y}}+\frac{(a-1) e^{-y} \dot{x} \dot{y}^{a-2} \ddot{y}}{\dot{y}^{2}+\ddot{y}} \\
\frac{\ddot{x}}{\dot{x} \dot{y}}=f(u, v), \frac{e^{-y} \ddot{x} \dot{y}^{a-1}}{\dot{y}^{2}+\ddot{y}}+\frac{(a-1) e^{-y} \dot{x} \dot{y}^{a-2} \ddot{y}}{\dot{y}^{2}+\ddot{y}}=g(u, v) \\
u=y, v=t \dot{y}, w=t^{(2 a-1) /(a-1)} \ddot{x}, \frac{d v}{d u}=1+\frac{t \ddot{y}}{\dot{y}} \\
t^{(2 a-1) /(a-1)} \ddot{x}=f(u, v), 1+\frac{t \ddot{y}}{\dot{y}}=g(u, v)\end{array}$ \\
\hline$A_{3,7}$ & 3 & $\begin{array}{l}u=y, v=\frac{\dot{y} e^{-a \arctan \dot{x}}}{\sqrt{1+\dot{x}^{2}}}, w=\frac{\ddot{x} e^{-a \arctan \dot{x}}}{\left(1+\dot{x}^{2}\right)^{3 / 2}}, \frac{d v}{d u}=\frac{-(a+\dot{x}) \ddot{x} e^{-a \arctan \dot{x}}}{\left(1+\dot{x}^{2}\right)^{3 / 2}}+\frac{\ddot{y} e^{-a \arctan \dot{x}}}{\dot{y} \sqrt{1+\dot{x}^{2}}} \\
\frac{\ddot{x} e^{-a \arctan \dot{x}}}{\left(1+\dot{x}^{2}\right)^{3 / 2}}=f(u, v), \frac{-(a+\dot{x}) \ddot{x} e^{-a \arctan \dot{x}}}{\left(1+\dot{x}^{2}\right)^{3 / 2}}+\frac{\ddot{y} e^{-a \arctan \dot{x}}}{\dot{y} \sqrt{1+\dot{x}^{2}}}=g(u, v) \\
u=y+\arctan \dot{x}, v=\frac{\dot{y} e^{-a \arctan \dot{x}}}{\sqrt{1+\dot{x}^{2}}}, w=\frac{\ddot{x} e^{-a \arctan \dot{x}}}{\left(1+\dot{x}^{2}\right)^{3 / 2}}, \\
\frac{d v}{d u}=\frac{-(a+\dot{x}) \dot{y} \ddot{x} e^{-a \arctan \dot{x}}}{\left(\ddot{x}+\left(1+\dot{x}^{2}\right) \dot{y}\right) \sqrt{1+\dot{x}^{2}}}+\frac{\ddot{y} \sqrt{1+\dot{x}^{2}} e^{-a \arctan \dot{x}}}{\ddot{x}+\left(1+\dot{x}^{2}\right) \dot{y}} \\
\frac{\ddot{x} e^{-a \arctan \dot{x}}}{\left(1+\dot{x}^{2}\right)^{3 / 2}}=f(u, v), \frac{-(a+\dot{x}) \dot{y} \ddot{x} e^{-a \arctan \dot{x}}}{\left(\ddot{x}+\left(1+\dot{x}^{2}\right) \dot{y}\right) \sqrt{1+\dot{x}^{2}}}+\frac{\ddot{y} \sqrt{1+\dot{x}^{2}} e^{-a \arctan \dot{x}}}{\ddot{x}+\left(1+\dot{x}^{2}\right) \dot{y}}=g(u, v) \\
u=y, v=\left(1+t^{2}\right) \dot{y}, w=\frac{\ddot{x} e^{a \arctan t}}{\dot{y}^{3 / 2}}, \frac{d v}{d u}=2 t+\frac{\left(1+t^{2}\right) \ddot{y}}{\dot{y}} \\
\frac{\ddot{x} e^{a \arctan t}}{\dot{y}^{3 / 2}}=f(u, v), 2 t+\frac{\left(1+t^{2}\right) \ddot{y}}{\dot{y}}=g(u, v)\end{array}$ \\
\hline$A_{3,8}$ & 3 & $\begin{array}{l}u=2 y-\dot{x}, v=\dot{x}^{2}-4 x \dot{y}, w=x \ddot{x}-2 x \dot{y}, \frac{d v}{d u}=-2 \dot{x}-\frac{4 x \ddot{y}}{2 \dot{y}-\ddot{x}} \\
x \ddot{x}-2 x \dot{y}=f(u, v),-2 \dot{x}-\frac{4 x \ddot{y}}{2 \dot{y}-\ddot{x}}=g(u, v) \\
u=y, v=\frac{\dot{x}}{(t-x)^{2} \dot{y}^{2}}, w=\frac{2}{(t-x) \dot{y}}+\frac{\ddot{y}}{\dot{y}^{2}}, \frac{d v}{d u}=\frac{\ddot{x}}{(t-x)^{2} \dot{y}^{3}}-\frac{2(1-\dot{x}) \dot{x} \dot{y}+2(t-x) \dot{x} \ddot{y}}{(t-x)^{3} \dot{y}^{4}} \\
\frac{2}{(t-x) \dot{y}}+\frac{\ddot{y}}{\dot{y}^{2}}=f(u, v), \frac{\ddot{x}}{(t-x)^{2} \dot{y}^{3}}-\frac{2(1-\dot{x}) \dot{x} \dot{y}+2(t-x) \dot{x}}{(t-x)^{3} \dot{y}^{4}}=g(u, v) \\
u=y, v=\frac{t \dot{x}-x}{\dot{y}}, w=\frac{\ddot{x}}{\dot{y}^{3}}, \frac{d v}{d u}=\frac{t \ddot{x}}{\dot{y}^{2}}-\frac{(t \dot{x}-x) \ddot{y}}{\dot{y}^{3}} \\
\frac{\ddot{x}}{\dot{y}^{3}}=f(u, v), \frac{t \ddot{x}}{\dot{y}^{2}}-\frac{(t \dot{x}-x) \ddot{y}}{\dot{y}^{3}}=g(u, v) \\
u=y, v=\frac{x \dot{y}}{\sqrt{1+\dot{x}^{2}}}, w=\frac{1+\dot{x}^{2}}{x^{3} \dot{y}^{3}}+\frac{\ddot{x}}{x^{2} \dot{y}^{3}}, \frac{d v}{d u}=\frac{\dot{x}\left(1+\dot{x}^{2}-x \ddot{x}\right)}{\left(1+\dot{x}^{2}\right)^{3 / 2}}+\frac{x \ddot{y}}{\dot{y} \sqrt{1+\dot{x}^{2}}} \\
\frac{1+\dot{x}^{2}}{x^{3} \dot{y}^{3}}+\frac{\ddot{x}}{x^{2} \dot{y}^{3}}=f(u, v), \frac{\dot{x}\left(1+\dot{x}^{2}-x \ddot{x}\right)}{\left(1+\dot{x}^{2}\right)^{3 / 2}}+\frac{x \ddot{y}}{\dot{y} \sqrt{1+\dot{x}^{2}}}=g(u, v)\end{array}$ \\
\hline
\end{tabular}


TABle 2: Continued.

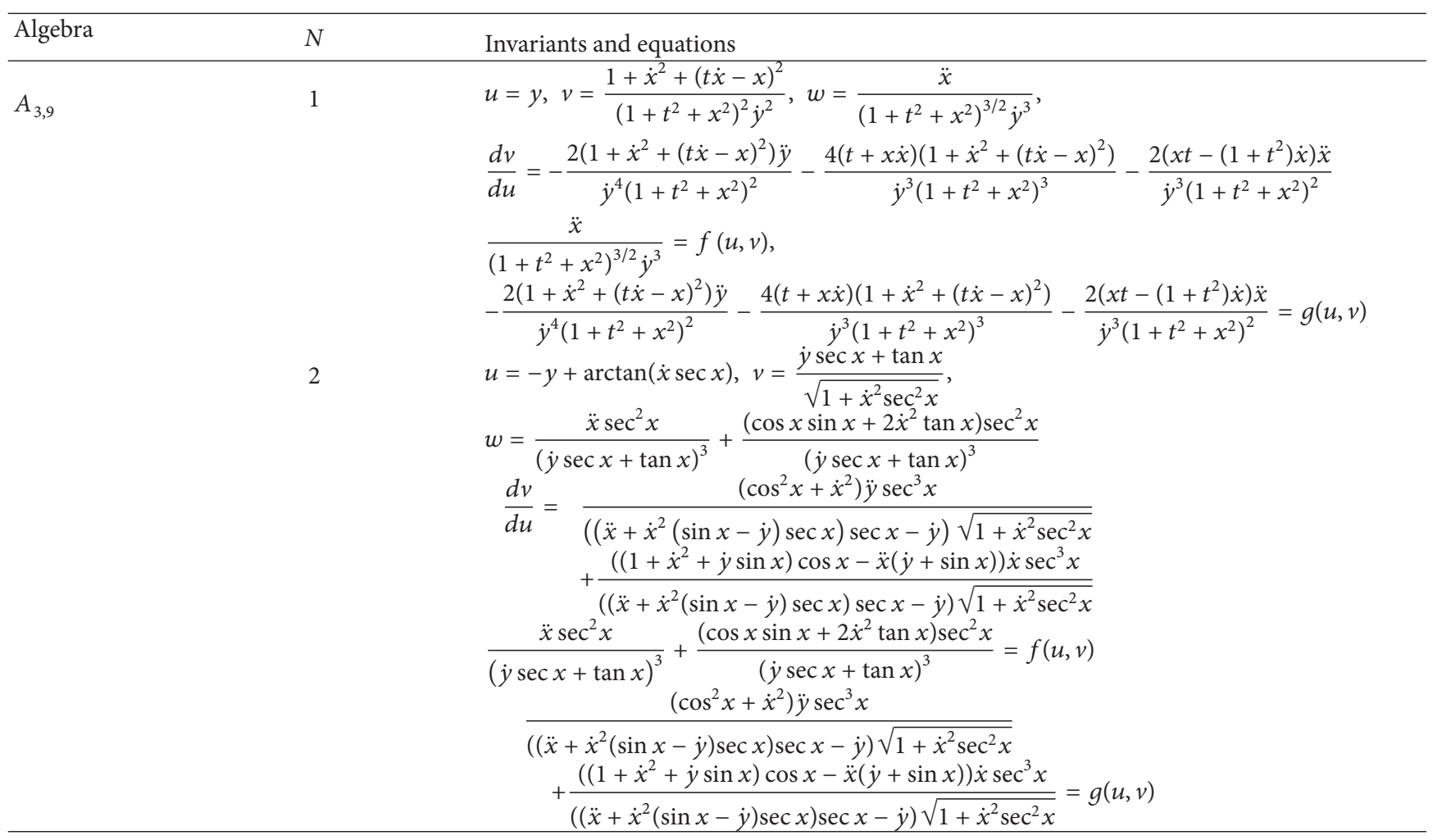

admitting the three-dimensional Lie algebra $A_{3,6}^{a, 2},|a| \leq$ $1, a \neq 0,1$. We arrive at the following set of invariants

$$
\begin{aligned}
& u=e^{y} \dot{y}, \quad v=\dot{x} \dot{y}^{a-1}, \quad w=\frac{\ddot{x}}{\dot{x} \dot{y}}, \\
& \frac{d v}{d u}=\frac{e^{-y} \ddot{x} \dot{y}^{a-1}}{\dot{y}^{2}+\ddot{y}}+\frac{(a-1) e^{-y} \dot{x} \dot{y}^{a-2} \ddot{y}}{\dot{y}^{2}+\ddot{y}} .
\end{aligned}
$$

This set involves three invariants $u, v$, and $w$ which play the role of basis of invariants for the considered system of secondorder ODEs admitting the three-dimensional Lie algebra $A_{3,6}^{a, 2},|a| \leq 1, a \neq 0,1$. This set is a complete set of invariants for the system. In the complete set, one of the invariants is obtained by differentiation. We state a result on invariant differentiation of invariants corresponding to a Lie algebra admitted by any system of ODEs which is already well-known for scalar ODEs in the literature.

Proposition 1. If $u$ and $v$ are invariants of a Lie algebra admitted by any system of ODEs, then, $d v / d u$ is also its invariant.

The proof is identical (except here we have many dependent variables) to the scalar case and uses the operator identity $X D_{t}-D_{t} X=-\left(D_{t} \xi\right) D_{t}$ (see, e.g., [17]).

One can write

$$
\frac{d v}{d u}=\frac{D_{t} v}{D_{t} u}=D v
$$

where the invariant differentiation operator is

$$
D=\left(D_{t} u\right)^{-1} D_{t}
$$

There arises a natural question. Can we construct a $k$ thorder system from a basis of invariants corresponding to the symmetry vectors of a second-order system? We answer this question.

Suppose that we have a complete set of invariants representing a second-order system of ODEs admitting a threedimensional Lie algebra, say for the above case $A_{3,6}^{a, 2},|a| \leq$ $1, a \neq 0,1$. Now if we differentiate both of the highestorder invariants from this set with respect to another basic invariant, we get two new invariants and the total number of invariants become six for this case, including the previous four. These two new invariants play the role of third-order invariants representing the third-order system of ODEs admitting the three-dimensional Lie algebra $A_{3,6}^{a, 2},|a| \leq$ $1, a \neq 0,1$. We therefore have for this case

$$
\begin{aligned}
& u=e^{y} \dot{y}, \quad v=\dot{x} \dot{y}^{a-1}, \quad w=\frac{\ddot{x}}{\dot{x} \dot{y}}, \\
& \frac{d v}{d u}=D v, \quad \frac{d w}{d u}=D w, \quad \frac{d^{2} v}{d u^{2}}=D^{2} v,
\end{aligned}
$$


where $D=e^{-y}\left(\dot{y}^{2}+\ddot{y}^{2}\right)^{-1} D_{t}$ and $D w$ and $D^{2} v$ are third-order invariants. We can thus construct the following third-order system

$$
\begin{gathered}
\frac{e^{-y} \ddot{x} \dot{x} \dot{y}-e^{-y} \ddot{x}(\dot{y} \ddot{x}+\dot{x} \ddot{y})}{\dot{x}^{2} \dot{y}^{2}\left(\dot{y}^{2}+\ddot{y}\right)}=f(u, v, w, D v), \\
\left(e ^ { - 2 y } \dot { y } ^ { a - 3 } \left(-\ddot{x} \dot{y}^{5}+(2 a-5) \ddot{x} \dot{y}^{3} \ddot{y}-(a-1) \dot{x} \dot{y}^{4} \ddot{y}\right.\right. \\
\left.\left.+2(a-1) \ddot{x} \dot{y} \ddot{y}^{2}\right)\right)\left(\left(\dot{y}^{2}+\ddot{y}\right)^{3}\right)^{-1} \\
+\left(e ^ { - 2 y } \dot { y } ^ { a - 3 } \left((a-1)\left((a-5) \dot{x} \dot{y}^{2} \ddot{y}^{2}+(a-2) \dot{x} \ddot{y}^{3}\right)\right.\right. \\
\left.\left.\quad+\dot{y}^{2} \ddot{x}\left(\dot{y}^{2}+\ddot{y}\right)+\dot{y}^{2}((a-1) \dot{x} \dot{y}-\ddot{x}) \ddot{y}\right)\right) \\
\times\left(\left(\dot{y}^{2}+\ddot{y}\right)^{3}\right)^{-1} \\
=g(u, v, w, D v) .
\end{gathered}
$$

We have constructed a system of two third-order ODEs admitting the three-dimensional Lie algebra $A_{3,6}^{a, 2},|a| \leq$ $1, a \neq 0,1$ from their complete set of invariants. This system of ODEs is represented by a complete set of invariants which contains six functionally independent invariants, namely, $u, v, w, D v, D w$, and $D^{2} v$, where three of them $u, v$, and $w$ are a basis of invariants and two of them $D w, D^{2} v$ are third-order invariants.

Now if we repeat this process on the third-order invariants, we obtain eight invariants including the previous ones which form a complete set of invariants for the fourth prolonged group. From these, we can construct a system of two fourth-order ODEs admitting the three-dimensional Lie algebra $A_{3,6}^{a, 2},|a| \leq 1, a \neq 0,1$. We can generalize this to a system of two $k$ th-order ODEs. However, there are some nonstandard cases to look at first. These behave differently. There are four cases $A_{3,1}^{3}, A_{3,5}^{3}, A_{3,1}^{4}$, and $A_{3,8}^{1}$ in which two of them $A_{3,1}^{3}$ and $A_{3,5}^{3}$ yield singular invariants and the other two $A_{3,1}^{4}$ and $A_{3,8}^{1}$ give a single equation and do not form a system.

2.1. Singular Case. In both of the cases $A_{3,1}^{3}$ and $A_{3,5}^{3}$, we have an extra condition, due to which the rank of the coefficient matrix on the solution manifold is less than the rank of the coefficient matrix on the generic manifold. So singular invariants are obtained in both of these cases.

Consider $A_{3,1}^{3} \simeq \partial_{x}, t \partial_{x}, y \partial_{x}$. We find the following set of singular invariants

$$
u=t, \quad v=y, \quad w=\ddot{x}, \quad D v=\dot{y}, \quad \ddot{y}=0,
$$

where $D=D_{t}$ and the corresponding system are

$$
\ddot{x}=f(u, v, D v), \quad \ddot{y}=0 .
$$

In both of the cases, the singularity of the invariants is removed in the third- and higher-order systems. In the case of $A_{3,1}^{3}$, we get the following complete set of invariants:

$$
\begin{gathered}
u=t, \quad v=y, \quad D v=\dot{y}, \\
D^{2} v=\ddot{y}, \quad w=\ddot{x} \ddot{y}-\ddot{x} \ddot{y}, \quad D^{3} v=\ddot{y},
\end{gathered}
$$

and the corresponding third-order system is

$$
\begin{gathered}
\ddot{x}=\frac{\ddot{x} \ddot{y}}{\ddot{y}}+\frac{1}{\ddot{y}} f\left(u, v, D v, D^{2} v\right), \\
\ddot{y}=g\left(u, v, D v, D^{2} v\right) .
\end{gathered}
$$

In this case, $u, v$, and $w$ form a basis of invariants, and here the third-order invariants are $w$ and $D^{3} v$, where $D=D_{t}$. For the other case $A_{3,5}^{3}$, it is easy to see that

$$
\begin{aligned}
& u=t, \quad v=y, \quad D v=\dot{y}, \\
& D^{2} v=\ddot{y}, \quad w=\frac{\ddot{x}}{\ddot{x}}, \quad D^{3} v=\ddot{y},
\end{aligned}
$$

where again $D=D_{t}$. For each of the remaining cases $A_{3,1}^{4}$ and $A_{3,8}^{1}$, we obtain the same set of second-order invariants

$$
\begin{aligned}
u=x, \quad v & =y, \quad D v=\frac{\dot{y}}{\dot{x}}, \\
D^{2} v & =\frac{\ddot{y}}{\dot{x}^{2}}-\frac{\dot{y} \ddot{x}}{\dot{x}^{3}},
\end{aligned}
$$

for which the highest-order invariant is only one and $D=$ $\dot{x}^{-1} D_{t}$. So a system of second-order ODEs cannot be constructed. Only a single scalar equation is obtained, namely

$$
\ddot{y}=\frac{\dot{y}}{\dot{x}} \ddot{x}+\dot{x}^{2} g(u, v, D v) .
$$

However, in both of these cases, this behavior changes for higher orders. In fact for $A_{3,1}^{4}$, we have the third-order invariants

$$
\begin{gathered}
w=\frac{\ddot{x} \phi^{\prime \prime}(x)}{\dot{x}^{4}}-\frac{3 \ddot{x}^{2} \phi^{\prime \prime}(x)+\dot{x}^{2} \ddot{x} \phi^{\prime \prime \prime}(x)}{\dot{x}^{5}}, \\
D^{3} v=\frac{\ddot{y}}{\dot{x}^{3}}+\frac{\left(3 \ddot{x}^{2}-\dot{x} \ddot{x}\right) \dot{y}-3 \dot{x} \ddot{x} \ddot{y}}{\dot{x}^{5}},
\end{gathered}
$$

and for $A_{3,8}^{1}$ the third-order invariants are

$$
w=\frac{\dddot{x}}{\dot{x}^{3}}-\frac{3 \ddot{x}^{2}}{2 \dot{x}^{4}}, \quad D^{3} v=\frac{\ddot{y}}{\dot{x}^{3}}+\frac{\left(3 \ddot{x}^{2}-\dot{x} \ddot{x}\right) \dot{y}-3 \dot{x} \ddot{x} \ddot{y}}{\dot{x}^{5}} .
$$

They give rise to a regular system of third-order ODE with a complete set of invariants having the same format as the singular cases similar to (13).

We find that, for a system of two second-order ODEs admitting three-dimensional Lie algebras, there are 29 classes 
which are listed in Table 2 together with their second-order differential invariants.

In the case of a system of two third- and higher-order ODEs admitting three-dimensional Lie algebras, there are 31 canonical forms, and these can be obtained by invariant differentiation as described above. Note here that $A_{3,1}^{4}$ and $A_{3,8}^{1}$ are admitted by a system of two third- and higher-order ODEs whereas they do not form a system of two second-order ODEs.

We state these results in the form of a theorem.

Theorem 2. A regular system of two kth-order ODEs $(k \geq 3)$ admitting three-dimensional Lie algebras can be represented by a complete set of invariants which contains $2 k$ complete functionally independent invariants in one of the following forms:

(a) $\quad u, v, w, \frac{d v}{d u}, \frac{d w}{d u}, \frac{d^{2} v}{d u^{2}}, \ldots, \frac{d^{k-2} w}{d u^{k-2}}, \frac{d^{k-1} v}{d u^{k-1}}$,

(b) $\quad u, v, \frac{d v}{d u}, \frac{d^{2} v}{d u^{2}}, w, \frac{d^{3} v}{d u^{3}}, \frac{d w}{d u}, \frac{d^{4} v}{d u^{4}}, \ldots, \frac{d^{k-3} w}{d u^{k-3}}, \frac{d^{k} v}{d u^{k}}$.

In both (a) and (b), $u, v$, and $w$ form a basis of invariants, where the highest order invariants in (a) are $d^{k-2} w / d u^{k-2}$, $d^{k-1} v / d u^{k-1}$ and in (b) they are $d^{k-3} w / d u^{k-3}, d^{k} v / d u^{k}$.

The total number of systems of two kth-order ODEs having the above structure is thirty-one, in which form (b) corresponds to the cases $A_{3,1}^{3}, A_{3,5}^{3}, A_{3,1}^{4}$, and $A_{3,8}^{1}$ and (a) to the remainder.

\section{Integrability}

There are basically two approaches to integrability using Lie point symmetries. One is that of successive reduction of order using differential invariants for scalar ODEs. The other is that of canonical forms which may apply to scalar as well as system of ODEs, although the latter is not straightforward. However, the canonical forms approach only applies when equations are classified according to the symmetry Lie algebras. In Table 2, we have given the complete set of second-order differential invariants of three-dimensional Lie algebras admitted by systems of two second-order ODEs and also classified systems of two second-order ODEs for their point symmetries together with their canonical forms. We provide two approaches for the integration procedure on the basis of these canonical forms. The first is a general approach depending on the subdivision of the canonical forms based on general observations. The second is the differential invariants approach based on the representation of the canonical forms in terms of first-order differential and algebraic invariants.

3.1. General Integration Approach. In this approach, we judiciously subdivide all the canonical forms into four types. They are as follows.

Type I:

$$
A_{3,1}^{3} \simeq \partial_{x}, t \partial_{x}, y \partial_{x}, \quad \ddot{x}=f(t, y, \dot{y}), \quad \ddot{y}=0 .
$$

Type II:

$$
A_{3,5}^{3}=\partial_{x}, t \partial_{x}, x \partial_{x}, \quad \ddot{y}=g(t, y, \dot{y}), \quad \ddot{x}=0 .
$$

Type III:

$$
\begin{gathered}
A_{3,2}^{4} \simeq \partial_{x}, t \partial_{t}+x \partial_{x}, t \partial_{x}, \\
t \ddot{x}=f(y, t \dot{y}), \quad 1+\frac{t \ddot{y}}{\dot{y}}=g(y, t \dot{y}), \\
A_{3,4}^{3} \simeq \partial_{x}, t \partial_{x},-\partial_{t}+x \partial_{x}, \\
e^{t} \ddot{x}=f(y, \dot{y}), \quad \frac{\ddot{y}}{\dot{y}}=g(y, \dot{y}), \\
A_{3,5}^{4} \simeq \partial_{x}, t \partial_{x}, x \partial_{x}+\partial_{y}, \\
e^{-y} \ddot{x}=f(t, \dot{y}), \quad \ddot{y}=g(t, \dot{y}), \\
A_{3,6}^{a, 3} \simeq \partial_{x}, t \partial_{x},(1-a) t \partial_{t}+x \partial_{x}, \\
t^{(2 a-1) /(a-1)} \ddot{x}=f(y, t \dot{y}), \quad 1+\frac{t \ddot{y}}{\dot{y}}=g(y, t \dot{y}), \\
A_{3,7}^{a, 3} \simeq \partial_{x}, t \partial_{x},-\left(1+t^{2}\right) \partial_{t}+(a-t) x \partial_{x}, \\
\ddot{x} \dot{y}^{-3 / 2} e^{a \tan t}=f\left(y, \dot{y}\left(1+t^{2}\right)\right), \\
2 t+\frac{\left(1+t^{2}\right)}{\dot{y}} \ddot{y}=g\left(y, \dot{y}\left(1+t^{2}\right)\right) .
\end{gathered}
$$

Type IV: Remaining canonical forms except those given above.

We notice that the Types I and II systems comprise linear equations. The Type I system is completely integrable. Also that the Type II system is uncoupled and depends on two quadratures of the $y$ equation for its solution. Further, we observe that the Type III has equations that comprise scalar second-order ODEs in the dependent variable $y$. In view of these observations, we are led to the following definitions.

Definition 3. A system of two second-order ODEs is said to be partially uncoupled if one of its equations constitutes a scalar second-order equation in one of the dependent variables and the other has both.

It is easily seen that the Types I to III equations are partially uncoupled.

Definition 4. A partially uncoupled system of two secondorder ODEs is partially linear if one of its equations forms a scalar linear second-order equation in its own right.

We see that the Types I and II systems are partially linear.

Definition 5. A system of two second-order ODEs is said to be partially linearizable by point transformation if it can be transformed via an invertible transformation to a partially linear form. 
The following system

$$
\ddot{x}=\dot{y}^{4}, \quad \ddot{y}+3 y \dot{y}+y^{3}=0
$$

is partially linearizable by a point transformation as the equation in $y$ is the well-known Painlevé-Ince equation which is linearizable via a point transformation.

Let $\left\{X_{i}\right\}$ be a basis of a three-dimensional algebra and let the rank of the coefficient matrix associated with these operators be $r$.

We formulate the following theorems the proofs of which are evident.

Theorem 6. A system of two second-order ODEs is partially linearizable by point transformation if and only if it admits the Lie algebra $A_{3,1}$ or $A_{3,5}$ with rank $r=1$ in both cases.

Theorem 7. A system of two second-order ODEs can be reduced to a partially uncoupled system by point transformation if and only if it admits one of the Lie algebras $A_{3,2}^{4}, A_{3,4}^{3}, A_{3,5}^{4}, A_{3,6}^{a, 3}$, and $A_{3,7}^{a, 3}$ with rank $r=2$ in all cases.

The proofs follow from the above listed Types I to III algebras and representative equations.

Generally, we can integrate Type I completely. Types II and III can be integrated when the associated secondorder equation in the system is integrable. Moreover, only particular cases of Type IV can be integrated depending on the given Lie algebra and corresponding system of ODEs. Some of these difficulties were also pointed out for systems of two second-order ODEs that admit four symmetries [12]. Here, we further discuss all the cases in detail before embarking on a further systematic study that captures the main results.

Type I is trivially integrable by quadratures.

The Type II system is integrable if the associated secondorder ODE in $y$ admits a two-dimensional Lie algebra and Lie's four canonical forms for scalar second-order ODEs become applicable. So, we have Lie reducibility here.

In the case of Type III, one requires one more symmetry for the associated equation in $y$ as it already has translations in $t$ symmetry. As an example we consider the system

$$
\ddot{x}=e^{-t} y^{3} \dot{y}^{4}, \quad \ddot{y}=\dot{y}^{2} y^{-1}-y^{2} .
$$

The system (25) has symmetry generators $X_{1}=\partial_{x}, X_{2}=$ $t \partial_{x}, X_{3}=-\partial_{t}+x \partial_{x}$ and its Lie algebra is three-dimensional. The associated equation in $y$ is $\ddot{y}=\dot{y}^{2} y^{-1}-y^{2}$. Its Lie algebra is two-dimensional spanned by $Y_{1}=\partial_{t}, Y_{2}=t \partial_{t}-2 y \partial_{y}$. By using the transformation

$$
\bar{t}=y, \quad \bar{x}=x, \quad \bar{y}=t,
$$

the associated equation in transformed form becomes $\ddot{\bar{y}}=$ $\bar{t}^{2} \dot{\bar{y}}^{3}-\dot{\bar{y}} \bar{t}^{-1}$. We have $\bar{Y}_{1}=\partial_{\bar{y}}, \bar{Y}_{2}=\bar{y} \partial_{\bar{y}}-2 \bar{t} \partial_{\bar{t}}$, which is Type III in Lie's classification of scalar second-order ODEs. Solving it in transformed form by Lie's method and reverting back to the original variables, we obtain $y=2 c_{1}^{2} \operatorname{sech}^{2} c_{1}\left(t-c_{2}\right)$, where $c_{1}$ and $c_{2}$ are constants. By using the value of $y$ in system (25) and then solving for $x$, we deduce

$$
\begin{aligned}
& x=2048 c_{1}{ }^{18} \int\left\{\int e^{-t} \operatorname{sech}^{14}\left(c_{1}\left(t-c_{2}\right)\right)\right. \\
&\left.\times \tanh ^{4}\left(c_{1}\left(t-c_{2}\right)\right) d t\right\} d t+c_{3} t+c_{4} .
\end{aligned}
$$

In general, the Type III systems of second-order ODEs are integrable if the associated second-order ODEs in $y$ have another symmetry generator besides translations in $t$, so that Lie's classification of scalar second-order ODEs admitting two-dimensional algebras is applicable here as well.

Theorem 8. If a singular system of two second-order ODEs admits the solvable symmetry algebra $A_{3,1}^{3}$, then, it is partially linearizable via point transformation and its general solution can be trivially obtained by quadratures.

The proof of this follows from the Type I canonical form.

Theorem 9. If a singular system of two second-order ODEs admits the three-dimensional solvable symmetry algebra $A_{3,5}^{3}$, then, it can be reduced to a partially uncoupled system and its general solution can be obtained by quadratures from the general solution of the associated transformed scalar secondorder ODE.

The proof here follows from the Type II canonical form.

For systems that fall in the Type IV category one requires more insight and the examples given in [12] for systems that admit four symmetries become relevant. Their integrability needs more than just the reductions via invariants. This category also has the nonsolvable algebras. We consider this in detail next.

3.2. Differential Invariant Approach. In this approach, we use the basis of invariants representing all the canonical forms which we have given in Table 2. For each regular canonical form, we find the reduced system in terms of a first-order equation and an algebraic equation based on the basis of invariants. These can easily be seen from Table 2 .

We have subdivided all the reduced systems in two main categories, namely, Category $A$ for those admitting threedimensional solvable Lie algebras and Category $B$ for those admitting three-dimensional nonsolvable Lie algebras. The Types I to III of the previous subsection all fall in Category $A$. The systems admitting nonsolvable algebras comprise Category B with the rest firmly falling in Category A. It is interesting that, in both categories, uncoupled cases arise when the choice of taking one of the second-order invariant $w$ is unique.

We present the integration strategy for Category $A$ for regular systems. The systems of Category $B$ are not amenable to such integration strategies. This is quite straightforward to work out if one commences with the simplest system in this category. We further subdivide Category A into coupled and uncoupled systems. All partially linear cases fall in the 
uncoupled systems. Here, we give details of integration for each case of Category $A$, which depends on the assumption that we can solve $d v / d u=h$ in both the coupled and the uncoupled systems. At the end of this section, we present some physical examples with detailed calculations.

Note here that, in both the uncoupled and coupled cases, for ease and simplicity of the calculations, we only mention the relevant algebra of the considered system and variables $u, v$, and $w$ as well as $d v / d u$ in precise form. The details are given in Table 2. In the following, we assume that $v=$ $\lambda\left(u, c_{1}\right)$ is the explicit solution of each of the first order ODEs $d v / d u=h(u, v)$ listed in Table 2. The argument presented below is similar to that used in the discussion of reduction to quadrature for the scalar ODEs (see [1-3]). Moreover, $f, g, h, k, \phi, \psi$, and $\lambda$ are taken as arbitrary functions and $c_{1}, c_{2}$, and $c_{3}$ as well as $c_{4}$ are arbitrary integration constants.

\subsubsection{Uncoupled Cases}

$A_{3,2}^{4}$. Let $v=\lambda\left(u, c_{1}\right)$. Then by using the values of $u$ and $v$ in Table 2, we have

$$
t \dot{y}=\lambda\left(y, c_{1}\right) .
$$

Thus, we can obtain a further quadrature in $y$. Therefore, the equation $t \ddot{x}=f(y, t \dot{y})$ is solvable by two quadratures upon insertion of the $y$ solution.

$A_{3,3}^{1}$. Let $v=\lambda\left(u, c_{1}\right)$. Then by invoking the values of $u$ and $v$ as listed in Table 2, we obtain

$$
\dot{x}=\dot{y} \lambda\left(y, c_{1}\right) .
$$

After some manipulations with $w=f(u, v)$, we find

$$
\dot{x}=\frac{-1}{\int \lambda\left(y, c_{1}\right) f\left(y, \lambda\left(y, c_{1}\right)\right) d y+c_{2}} .
$$

By using (30) in (29), we determine

$$
\dot{y} \lambda\left(y, c_{1}\right)\left\{\int \lambda\left(y, c_{1}\right) f\left(y, \lambda\left(y, c_{1}\right)\right) d y+c_{2}\right\}=-1,
$$

which implies that

$$
y=\phi\left(t, c_{1}, c_{2}, c_{3}\right) .
$$

Utilizing (32) in (30), after some further calculations, we deduce

$$
x=\psi\left(t, c_{1}, c_{2}, c_{3}, c_{4}\right) .
$$

Hence, (32) and (33) are the solutions of the system of ODEs admitting $A_{3,3}^{1}$.

$A_{3,3}^{2}$. Let $v=\lambda\left(u, c_{1}\right)$. By using the values of $u$ and $v$ as listed in Table 2, we arrive at

$$
\dot{x}= \pm \sqrt{2 \dot{y}+2 \dot{y}^{2} \lambda\left(y, c_{1}\right)} .
$$

After calculations with $w=f(u, v)$, we find

$$
\dot{x}=-\dot{y}\left\{\int f\left(y, \lambda\left(y, c_{1}\right)\right) d y+c_{2}\right\} .
$$

Invoking (35) in (34) and solving for $\dot{y}$, we obtain

$$
\dot{y}=\frac{2}{\left(\int f\left(y, \lambda\left(y, c_{1}\right)\right) d y+c_{2}\right)^{2}-2 \lambda\left(y, c_{1}\right)},
$$

which implies that

$$
y=\phi\left(t, c_{1}, c_{2}, c_{3}\right)
$$

After some computations and using (37) in (35), we determine

$$
x=\psi\left(t, c_{1}, c_{2}, c_{3}, c_{4}\right) .
$$

It is hence the case that (37) and (38) are the solutions of the system of ODEs corresponding to $A_{3,3}^{2}$.

$A_{3,4}^{3}$. Let $v=\lambda\left(u, c_{1}\right)$. Using the values of $u$ and $v$, we obtain

$$
\dot{y}=\lambda\left(y, c_{1}\right)
$$

which implies that

$$
y=\phi\left(t, c_{1}, c_{2}\right)
$$

Now utilizing (39) and (40) in $w=f(u, v)$, we deduce that

$$
x=\int\left\{\int e^{-t} f\left(\phi, \phi_{t}\right) d t\right\} d t+c_{3} t+c_{4}
$$

which results in

$$
x=\psi\left(t, c_{1}, c_{2}, c_{3}, c_{4}\right) .
$$

Now (40) and (42) are the solutions of the system of ODEs possessing $A_{3,4}^{3}$.

$A_{3,5}^{1}$. Let $v=\lambda\left(u, c_{1}\right)$. Utilizing the values of $u$ and $v$ as listed, we obtain

$$
\dot{x}=\lambda\left(y, c_{1}\right) .
$$

After some manipulations with $w=f(u, v)$, we find

$$
\int \frac{d y}{c_{2} \exp \left\{\int f\left(y, \lambda\left(u, c_{1}\right)\right) d y\right\}}=t+c_{3} .
$$

Inverting, we have

$$
y=\phi\left(t, c_{1}, c_{2}, c_{3}\right)
$$

By using (45) in (43), we have that

$$
x=\psi\left(t, c_{1}, c_{2}, c_{3}, c_{4}\right) .
$$

Thus, (45) and (46) are the solutions of the system of ODEs corresponding to $A_{3,5}^{1}$.

For $A_{3,5}^{4}, A_{3,6}^{a, 3}$, and $A_{3,7}^{a, 3}$, the integration procedure works the same as in the case $A_{3,4}^{3}$. 


\subsubsection{Coupled Cases}

$A_{3,1}^{1}$. Let $v=\lambda\left(u, c_{1}\right)$. Using the values of $u$ and $v$ as listed in Table 2, we obtain

$$
\dot{y}=\lambda\left(\dot{x}, c_{1}\right) .
$$

After calculations with $w=f(u, v)$, we arrive at

$$
x=\phi\left(t, c_{1}, c_{2}, c_{3}\right) .
$$

By utilizing (48) in (47), in the same manner as in the previous cases, we find that

$$
y=\psi\left(t, c_{1}, c_{2}, c_{3}, c_{4}\right) .
$$

Hence (48) and (49) are the solutions of the system of ODEs corresponding to $A_{3,1}^{1}$.

$A_{3,1}^{2}$. The procedure of integration is the same as in the case $A_{3,1}^{1}$.

$A_{3,2}^{1}$. Let $v=\lambda\left(u, c_{1}\right)$. Using the values of $u$ and $v$ as listed in Table 2, we determine

$$
\dot{x}=\dot{y} \lambda\left(\dot{y} e^{y}, c_{1}\right) .
$$

After some manipulations with $w=f(u, v)$, we find

$$
\begin{aligned}
\dot{x}=\exp \left\{\int\right. & \left(\left(\lambda\left(\dot{y} e^{y}, c_{1}\right)+\dot{y} e^{y} g\left(\dot{y} e^{y}, \lambda\left(\dot{y} e^{y}, c_{1}\right)\right)\right)\right. \\
& \left.\times f\left(\dot{y} e^{y}, \lambda\left(\dot{y} e^{y}, c_{1}\right)\right) d\left(\dot{y} e^{y}\right)\right) \\
\times & \left.\left(\dot{y} e^{y}\left(1+\lambda\left(\dot{y} e^{y}, c_{1}\right) f\left(\dot{y} e^{y}, \lambda\left(\dot{y} e^{y}, c_{1}\right)\right)\right)\right)^{-1}\right\} .
\end{aligned}
$$

From the second equation of the system in Table 2 and some manipulations with (50), we find that

$$
\begin{aligned}
& \frac{d\left(\dot{y} e^{y}\right)}{d t} \\
& \quad=\frac{\dot{y}^{2} e^{y} \lambda\left(\dot{y} e^{y}, c_{1}\right)\left(1+\lambda\left(\dot{y} e^{y}, c_{1}\right) f\left(\dot{y} e^{y}, \lambda\left(\dot{y} e^{y}, c_{1}\right)\right)\right)}{\left(\lambda\left(\dot{y} e^{y}, c_{1}\right)+\dot{y} e^{y} g\left(\dot{y} e^{y}, \lambda\left(\dot{y} e^{y}, c_{1}\right)\right)\right)} .
\end{aligned}
$$

Some further computations give

$$
\dot{y} e^{y}=\phi\left(y+c_{2}\right) \text {, }
$$

which implies that

$$
y=h\left(t, c_{1}, c_{2}, c_{3}\right)
$$

Using (53) and (54) in (51), we find

$$
x=k\left(t, c_{1}, c_{2}, c_{3}, c_{4}\right) .
$$

Therefore, (54) and (55) are the solutions of the system of ODEs corresponding to $A_{3,2}^{1}$.
$A_{3,2}^{2}$. The procedure of integration is the same as the case $A_{3,5}^{1}$. $A_{3,2}^{3}$. The procedure of integration is similar to the case $A_{3,3}^{1}$. $A_{3,3}^{3}$. Let $v=\lambda\left(u, c_{1}\right)$. Using the values of $u$ and $v$ as listed in Table 2, we obtain

$$
\frac{\dot{x}}{\dot{y}}=\lambda\left(y-\frac{1}{\dot{x}}, c_{1}\right) .
$$

Using the chain rule and some manipulations with $w=$ $f(u, v)$, we arrive at

$$
y=\phi\left(y-\frac{1}{\dot{x}}, c_{1}, c_{2}\right) .
$$

Further simplifications give

$$
\dot{x}=\frac{1}{y-\phi^{-1}\left(y, c_{1}, c_{2}\right)} .
$$

Utilizing (57) and (58) in (56), we find that

$$
\dot{y}\left(y-\phi^{-1}\left(y, c_{1}, c_{2}\right)\right) \lambda\left(\phi^{-1}\left(y, c_{1}, c_{2}\right), c_{1}\right)=1,
$$

which implies that

$$
y=h\left(t, c_{1}, c_{2}, c_{3}\right)
$$

Invoking (60) in (58), we have

$$
x=k\left(t, c_{1}, c_{2}, c_{3}, c_{4}\right) .
$$

Thus, (60) and (61) are the solutions of the system of ODEs possessing $A_{3,3}^{3}$.

$A_{3,4}^{1}$. The procedure of integration is similar to the case $A_{3,3}^{1}$. $A_{3,4}^{2}$. The procedure of integration is the same as the case $A_{3,3}^{3}$. $A_{3,5}^{2}$. Let $v=\lambda\left(u, c_{1}\right)$. Utilizing the values of $u$ and $v$ as listed in Table 2, we obtain

$$
\dot{y} e^{y}=\lambda\left(\dot{x}, c_{1}\right) \text {. }
$$

By the chain rule and some simplifications with $w=f(u, v)$, we find

$$
\dot{x}=\phi\left(y, c_{1}, c_{2}\right) .
$$

Substituting (63) in (62) and further manipulations give

$$
y=\psi\left(x, c_{1}, c_{2}, c_{3}\right) .
$$

Invoking this in (63), we deduce that

$$
x=h\left(t, c_{1}, c_{2}, c_{3}, c_{4}\right) .
$$

Now after substitution of (65), (64) takes the form

$$
y=\psi\left(t, c_{1}, c_{2}, c_{3}, c_{4}\right) .
$$

Here, (65) and (66) are the solutions of the system of ODEs corresponding to $A_{3,5}^{2}$. 
$A_{3,6}^{a, 1}$. The procedure of integration is the same as the case $A_{3,3}^{1}$. $A_{3,6}^{a, 2}$. The procedure of integration is again similar to the case $A_{3,2}^{1}$.

$A_{3,7}^{a, 1}$. The procedure of integration corresponds to the case $A_{3,3}^{1}$ and some details are given in the case $A_{3,7}^{a, 2}$.

$A_{3,7}^{a, 2}$. Let $v=\lambda\left(u, c_{1}\right)$. Using the values of $u$ and $v$ as listed in Table 2, we obtain

$$
\frac{\dot{y} e^{-a \arctan \dot{x}}}{\sqrt{1+\dot{x}^{2}}}=\lambda\left(y+\arctan \dot{x}, c_{1}\right) .
$$

After manipulations with $w=f(u, v)$, we find

$$
\dot{x}=\tan \left\{\int \frac{f\left(u, \lambda\left(u, c_{1}\right)\right) d u}{\lambda\left(u, c_{1}\right)+f\left(u, \lambda\left(u, c_{1}\right)\right)}+c_{2}\right\} .
$$

Invoking (68) in (67), we determine

$$
\begin{aligned}
\dot{y}= & \lambda\left(u, c_{1}\right) \sec a\left\{\int \frac{f\left(u, \lambda\left(u, c_{1}\right)\right) d u}{\lambda\left(u, c_{1}\right)+f\left(u, \lambda\left(u, c_{1}\right)\right)}+c_{2}\right\} \\
& \times \exp \left\{a \int \frac{f\left(u, \lambda\left(u, c_{1}\right)\right) d u}{\lambda\left(u, c_{1}\right)+f\left(u, \lambda\left(u, c_{1}\right)\right)}+a c_{2}\right\} .
\end{aligned}
$$

We have

$$
\frac{d u}{d t}=\frac{\dot{y}\left(\lambda\left(u, c_{1}\right)+f\left(u, \lambda\left(u, c_{1}\right)\right)\right)}{\lambda\left(u, c_{1}\right)} .
$$

In $(68)-(70)$,

$$
u=y+\arctan \dot{x} .
$$

Also, we find that

$$
\begin{gathered}
u=\phi^{-1}\left(t+c_{3}\right), \\
y+\arctan \dot{x}=\phi^{-1}\left(t+c_{3}\right) .
\end{gathered}
$$

Using (69) and (72) in (70), we deduce

$$
u=\psi\left(t, c_{1}, c_{2}, c_{3}\right)
$$

which shows that

$$
y=h\left(t, c_{1}, c_{2}, c_{3}\right)
$$

By invoking (74) in (68), we have that

$$
x=k\left(t, c_{1}, c_{2}, c_{3}, c_{4}\right) .
$$

Thus, (74) and (75) are the solutions of the system of ODEs corresponding to $A_{3,7}^{a, 2}$.

In the view of the preceding reductions, we have the following theorem.

Theorem 10. If a regular system of two second-order ODEs admits a three-dimensional solvable symmetry algebra, then, the general solution of the regular system can be obtained by quadratures from the general solution of the invariant representation of the regular system given by the first-order ordinary differential equation and algebraic equation.
Theorem 11. If a system of two kth-order $(k \geq 3)$ ODEs possess a three-dimensional solvable symmetry algebra, then, the general solution of the system can be deduced by quadratures from the general solution of the invariant representation given by one of the following systems:

(a) $\frac{d^{k-1} v}{d u^{k-1}}=f\left(u, v, w, \frac{d v}{d u}, \frac{d w}{d u}, \frac{d^{2} v}{d u^{2}}, \ldots, \frac{d^{k-3} w}{d u^{k-3}}, \frac{d^{k-2} v}{d u^{k-2}}\right)$,

$$
\frac{d^{k-2} w}{d u^{k-2}}=g\left(u, v, w, \frac{d v}{d u}, \frac{d w}{d u}, \frac{d^{2} v}{d u^{2}}, \ldots, \frac{d^{k-3} w}{d u^{k-3}}, \frac{d^{k-2} v}{d u^{k-2}}\right),
$$

(b) $\frac{d^{k} v}{d u^{k}}$

$$
=f\left(u, v, \frac{d v}{d u}, \frac{d^{2} v}{d u^{2}}, w, \frac{d^{3} v}{d u^{3}}, \frac{d w}{d u}, \frac{d^{4} v}{d u^{4}}, \ldots, \frac{d^{k-4} w}{d u^{k-4}}, \frac{d^{k-1} v}{d u^{k-1}}\right),
$$

$\frac{d^{k-3} w}{d u^{k-3}}$

$$
=g\left(u, v, \frac{d v}{d u}, \frac{d^{2} v}{d u^{2}}, w, \frac{d^{3} v}{d u^{3}}, \frac{d w}{d u}, \frac{d^{4} v}{d u^{4}}, \ldots, \frac{d^{k-4} w}{d u^{k-4}}, \frac{d^{k-1} v}{d u^{k-1}}\right) .
$$

The proof follows from Theorem 2 and the discussion preceding Theorem 10.

3.2.3. Illustrative Examples. Here, we present some familiar physical examples to illustrate the above general integration procedure which we have given in detail for each of the case of system of ODEs admitting solvable three-dimensional Lie algebras. We consider two different cases, namely, one from the uncoupled cases and one from the coupled cases. We also present a case of nonsolvable Lie algebras. Here, we observe interesting results rather than integration.

(i) Consider the system (25) once again from a new angle. We have

$$
\ddot{x}=e^{-t} y^{3} \dot{y}^{4}, \quad \ddot{y}=\dot{y}^{2} y^{-1}-y^{2} .
$$

The system (25) has symmetry generators $X_{1}=\partial_{x}, X_{2}=$ $t \partial_{x}, X_{3}=-\partial_{t}+x \partial_{x}$ and its Lie algebra is three-dimensional which is $A_{3,4}^{3}$ in our classification. From Table 2, the firstorder differential invariants for this case are

$$
u=y, \quad v=\dot{y},
$$

and the reduced system of first-order equation and algebraic equation is

$$
\frac{d v}{d u}=g(u, v), \quad w=f(u, v)
$$

The solution of $d v / d u$ in system (79), after substituting the value of $g$ from (25), gives

$$
v= \pm u \sqrt{2\left(c_{1}^{*}-u\right)}
$$


where $c_{1}^{*}$ is constant. Reverting back to the original variables and after solving for $y$, we obtain

$$
y=2 c_{1}^{2} \operatorname{sech}^{2} c_{1}\left(t-c_{2}\right),
$$

where $c_{1}=\sqrt{c_{1}^{*} / 2}$ and $c_{2}$ are constants. Substituting the values in $w$ and solving for $x$, we find

$$
\begin{aligned}
x=2048 c_{1}{ }^{18} \int\left\{\int\right. & e^{-t} \operatorname{sech}^{14}\left(c_{1}\left(t-c_{2}\right)\right) \\
& \left.\times \tanh ^{4}\left(c_{1}\left(t-c_{2}\right)\right) d t\right\} d t+c_{3} t+c_{4} .
\end{aligned}
$$

Both (81) and (82) provide the solution of system (25).

(ii) The classical Kepler problem in reduced Cartesian coordinates is

$$
\ddot{\vec{r}}+\mu \frac{\vec{r}}{r^{3}}=0,
$$

where $\mu$ is a constant. In polar coordinates, they are

$$
\ddot{r}-r \dot{\theta}^{2}+\mu r^{-2}=0, \quad r \ddot{\theta}+2 \dot{r} \dot{\theta}=0 .
$$

It has the three-dimensional Lie algebra of symmetries [18] spanned by

$$
X_{1}=\partial_{t}, \quad X_{2}=\partial_{\theta}, \quad X_{3}=t \partial_{t}-\frac{2}{3} r \partial_{r} .
$$

By using the transformation

$$
\bar{t}=t, \quad \bar{x}=\theta, \quad \bar{y}=\frac{3}{2} \ln r
$$

the system (84) transforms into

$$
\ddot{\bar{y}}+\frac{2}{3} \dot{\bar{y}}^{2}-\frac{3}{2} \dot{\bar{x}}^{2}+\frac{3}{2} \mu e^{-2 \dot{\bar{y}}}=0, \quad \frac{\ddot{x}}{3}+\frac{4}{3} \dot{\bar{x}} \dot{\bar{y}}=0,
$$

with associated transformed Lie algebra of symmetries

$$
X_{1}=\partial_{\bar{t}}, \quad X_{2}=\partial_{\bar{x}}, \quad X_{3}=\bar{t} \partial_{\bar{t}}+\partial_{\bar{y}},
$$

which is $A_{3,2}^{1}$ in our classification. From Table 2, the firstorder differential invariants for this case are

$$
u=\dot{\bar{y}} e^{\bar{y}}, \quad v=\frac{\dot{\bar{x}}}{\dot{\bar{y}}},
$$

and the reduced system of first-order equation and algebraic equation is

$$
\frac{d v}{d u}=g(u, v), \quad w=f(u, v) .
$$

By utilizing the values of $f$ and $g$ from (87), we have that

$$
\frac{d v}{d u}=\frac{-4 v+9\left(\mu v / u^{2}\right)-9 v^{3}}{2 u-9(\mu / u)+9 v^{2} u}, \quad w=-\frac{4}{3 v} .
$$

Now the solution of $d v / d u$ in the system (91) is

$$
u^{4} v^{2}-\frac{9}{2} \mu u^{2} v^{2}+\frac{9}{4} u^{4} v^{4}=h
$$

In $\bar{x}$ and $\bar{y}$ variables, after some simplification, (92) and $w$ from (91) give

$$
\begin{gathered}
\frac{d\left(e^{(2 / 3) \bar{y}}\right)}{d t}= \pm \sqrt{\frac{4 h}{9 k^{2}}+2 \mu e^{(2 / 3) \bar{y}}-e^{(4 / 3) \bar{y}},} \\
\left(e^{(2 / 3) \bar{y}}\right)^{2} \dot{\bar{x}}=k,
\end{gathered}
$$

where $h$ and $k$ are constants. Reverting back to the original variables, we deduce

$$
\begin{gathered}
\frac{d r}{d t}= \pm \sqrt{\frac{4 h}{9 k^{2}}+\frac{2 \mu}{r}-\frac{1}{r^{2}}} \\
r^{2} \dot{\theta}=k .
\end{gathered}
$$

The term on the left-hand side of (95) is the angular momentum and here it is constant from the calculations and denoted by $L / m$. Performing a simple integration procedure, we arrive at the equation of the orbit, namely,

$$
d \theta=\frac{d s}{\mp \sqrt{\left(h^{*}+2 m^{2} \mu s / L^{2}-s^{2}\right)}},
$$

where $s(\theta)=1 / r(\theta)$ and $h^{*}=4 h / 9 k^{4}$. This corresponds to that presented in [19].

(iii) Consider a case of nonsolvable Lie algebras, namely, the generalized Ermakov system in polar coordinates of the form

$$
\begin{aligned}
& \ddot{r}-r \dot{\theta}^{2}=\frac{1}{r^{3}} f\left(\theta, r^{2} \dot{\theta}\right), \\
& r \ddot{\theta}+2 \dot{r} \dot{\theta}=\frac{1}{r^{3}} g\left(\theta, r^{2} \dot{\theta}\right),
\end{aligned}
$$

which was considered in [20]. This system has the three symmetries

$$
X_{1}=\partial_{t}, \quad X_{2}=2 t \partial_{t}+r \partial_{r}, \quad X_{3}=t^{2} \partial_{t}+t r \partial_{r},
$$

and forms the Lie algebra $\mathrm{sl}(2, R)$ which is $A_{3,8}^{4}$ in our classification. The first-order differential invariants for this case are $u=y, v=(t \dot{x}-x) / \dot{y}$, and the reduced system of first-order equation and an algebraic equation is $d v / d u=$ $g(u, v), w=f(u, v)$. By using these and considering the angular momentum as constant, $L=m r^{2} \dot{\theta}$, we find that $d v / d u=-v h(u, L / m)$, where $g(u, v)=-v h(u, L / m)$. In the original variables, it is

$$
M=\frac{1}{2}\left(r^{2} \dot{\theta}\right)^{2}-\int h(\theta) d \theta,
$$

where $h(\theta)=h(u, L / m)$. Here $M$ is the Ermakov invariant. The classical integrability was discussed in [20]. 


\section{Conclusion}

The integrability of systems of ordinary differential equations (ODEs) which admit symmetry algebras has been of interest in the literature. The traditional symmetry reductions do not work for systems of ODEs as in the case of scalar ODEs (see, e.g., $[5,12,17])$. Here, we have focused on systems of two $k$ th-order $(k \geq 2)$ ODEs that admit three-dimensional Lie algebras.

By utilizing the realizations given recently, we have provided a systematic approach for finding a complete set of differential invariants including basis of invariants corresponding to vector fields in three variables for systems of two $k$ th-order $(k \geq 2)$ ODEs which admit three-dimensional Lie algebras. In particular, we presented a complete list of secondorder differential invariants for systems of two second-order ODEs that admit three-dimensional Lie algebras. We have shown how invariant differentiations provide higher-order differential invariants. We have given a complete list of invariant characterizations of canonical forms for systems of two second-order ODEs that admit real Lie algebras of dimension three. These are new in the literature. In addition, we have given a procedure for the construction of two $k$ thorder ODEs from their associated complete set of invariants. We have shown that there are 29 classes for the case of $k=2$ and 31 classes for the case of $k \geq 3$ of such ODEs. Furthermore, we have given a discussion of those cases in which regular systems of two second-order ODEs are not obtained for three-dimensional Lie algebras. We also have given a brief discussion on the singularity for canonical forms for a system of two second-order ODEs admitting-three dimensional Lie algebras and found two cases of singular invariants, namely, those involving the algebras $A_{3,1}^{3}$ and $A_{3,5}^{3}$.

We have provided an integration procedure for the canonical forms for a system of two second-order ODEs which possess three-dimensional Lie algebras. This procedure is composed of two approaches, namely, one which depends on general observations of canonical forms; the other depends on basis of differential invariants and the solution procedure is applicable only for those cases which admit three-dimensional solvable Lie algebras. The latter was extended in a natural way to $k$ th-order $(k \geq 3)$ systems that admit three-dimensional Lie algebras. Specifically in the second approach, we obtain the reducibility for canonical forms for systems of two second-order ODEs which have three-dimensional Lie algebras in terms of basis of invariants; these are given in Table 2. Then, we gave details of integration for each case of solvable Lie algebras in a general manner.

We also have obtained two cases of partial linearization, namely, Types I and II. It was noted that the Type I system is trivially integrable. We have further shown how the associated scalar second-order ODEs in the Types II to III can be solved in terms of quadratures resorting to the classical Lie table for integrability of scalar ODEs. This was illustrated by means of an example. There are certain Type IV systems which have complications in their integrability in that they admit nonsolvable algebras.

We have presented familiar physical systems taken from the literature, namely, the classical Kepler problem and the generalized Ermakov systems that give rise to closed trajectories.

It would be of interest to further pursue integrability properties of Type IV systems having nonsolvable Lie algebras. Also worthy of investigation are systems that admit higher number of symmetries such as those studied in $[12,16]$. We return to these in the future.

\section{Remarks}

(i) In the tables, $A$ is used as a place holder for the relevant real Lie algebras $A_{i, j}^{a, b, n} \ldots$ (the $j$ th algebra of dimension $i$ with superscripts $a, b$, if any, indicates parameters on which the Lie algebra depends; also the column $N$ in the tables gives the algebra realizations; the realization is referred to by a superscript $n$, e.g., $\left.A_{3,1}^{1}\right)$. Further we use the notation $\partial_{t}=$ $\partial / \partial t, \partial_{x}=\partial / \partial x, \partial_{y}=\partial / \partial y$. Finally, the elements of a basis of a given Lie algebra are named $X_{i}$, where $i$ is less than or equal to the dimension of the underlying real Lie algebra. Here, $f, g, h, k$, and $\phi$ are arbitrary functions.

(ii) For the analysis of the invariant representations, it is observed that for each of the cases $A_{3,1}^{4}$ and $A_{3,8}^{1}$, with the same set of invariants, a single equation is determined. We find the differential invariants in both cases. However, the canonical form is not obtained. Consider, for example, $A_{3,1}^{4} \simeq$ $\partial_{t}, x \partial_{t}, \phi(x) \partial_{t}, \quad \phi^{\prime \prime}(x) \neq 0$. For this, we find the following invariants $u=x, v=y, d v / d u=\dot{y} / \dot{x}$, $d^{2} v / d u^{2}=-\ddot{x} \dot{y} / \dot{x}^{3}+\ddot{y} / \dot{x}^{2}$ and the corresponding single equation, namely, $-\ddot{x} \dot{y} / \dot{x}^{3}+\ddot{y} / \dot{x}^{2}=g(u, v, d v / d u)$. For each of the cases $A_{3,1}^{3}$ and $A_{3,5}^{3}$, due to the extra condition, the rank of the coefficient matrix on the solution manifold is less than the rank of generic coefficient matrix. So only a set of singular invariants are obtained for these cases. Consider, for example, $A_{3,1}^{3} \simeq \partial_{x}, t \partial_{x}, y \partial_{x}$. For this, we find the following set of singular invariants $u=t, v=y, d v / d u=$ $\dot{y}, w=\ddot{x}$, and $d v / d u=\ddot{y}=0$ (see Table 2). The two cases $A_{3,1}^{4}$ and $A_{3,8}^{1}$ are not given in the invariant representations listed in Table 2 .

(iii) The algebras $A_{3,1}^{3}$ and $A_{3,5}^{3}$ which are referred to by * give rise to singular invariants when associated with systems of two second-order ODEs as given in Table 2. We do however arrive at regular invariants associated with systems of two third-order ODEs for these cases.

(iv) The algebras $A_{3,1}^{4}$ and $A_{3,8}^{1}$ do not correspond to systems of two second-order ODEs and so are not presented in Table 2.

\section{Acknowledgments}

M. Ayub and M. Khan are grateful to the Higher Education Commission (HEC) of Pakistan for financial support (via 
Indigenous Scholarship and IRSIP program). Also F. M. Mahomed thanks the HEC for granting him a visiting professorship and the Department of Mathematics at the Quaidi-Azam University, Islamabad, for its hospitality during the time this work was initiated. M. Ayub also thanks the Centre for Differential Equations, Continuum Mechanics and Applications, School of Computational and Applied Mathematics, University of the Witwatersrand, South Africa, for its hospitality during the time this project was completed. Finally F. M. Mahomed is grateful to the NRF of South Africa for research funding. Parts of this work was presented at the SDEA2012 Jan Conference.

\section{References}

[1] S. Lie, "Klassiffikation und Integration von gewönlichen Differentialgleichungen zwischen $x, y$, die eine Gruppe von Transformationen gestaten," Archiv der Mathematik, vol. 8, no. 9, p. 187, 1883.

[2] S. Lie, Vorlesungen Über Differentialgleichungen Mit Bekannten Infinitesimalen Transformationen, B. G. Teubner, Leipzig, Germany, 1891.

[3] F. M. Mahomed and P. G. L. Leach, "Lie algebras associated with scalar second-order ordinary differential equations," Journal of Mathematical Physics, vol. 30, no. 12, pp. 2770-2777, 1989.

[4] F. M. Mahomed and P. G. L. Leach, "Symmetry Lie algebras of $n$th order ordinary differential equations," Journal of Mathematical Analysis and Applications, vol. 151, no. 1, pp. 80-107, 1990.

[5] C. Wafo Soh and F. M. Mahomed, "Reduction of order for systems of ordinary differential equations," Journal of Nonlinear Mathematical Physics, vol. 11, no. 1, pp. 13-20, 2004.

[6] R. O. Popovych, V. M. Boyko, M. O. Nesterenko, and M. W. Lutfullin, "Realizations of real low-dimensional Lie algebras," Journal of Physics, vol. 36, no. 26, pp. 7337-7360, 2003.

[7] S. Lie, Theories der Transformation Gruppen, Chelsea Publishing, New York, NY, USA, 2nd edition, 1970, Originally published in Leipzig, 1888, 1890, 1893.

[8] S. Lie, "Theorie der transformationsgruppen," Mathematische Annalen, vol. 16, no. 4, pp. 441-528, 1880.

[9] S. Lie, "Theorie der transformationsgruppen," in Gesammetle Abhandlungen, vol. 6, pp. 1-94, B. G. Teubner, Leipzig, Germany, 1927.

[10] L. Bianchi, Lezioni Sulla Teoria dei Gruppi Continui Finiti di Transformazioni, Enirco Spoerri, Pisa, Italy, 1918.

[11] G. M. Mubarakzjanov, “On solvable Lie algebras," Izvestija Vysših Učebnyh Zavedeniı̌ Matematika, vol. 1, no. 32, pp. 114123, 1963 (Russian).

[12] C. Wafo Soh and F. M. Mahomed, "Canonical forms for systems of two second-order ordinary differential equations," Journal of Physics, vol. 34, no. 13, pp. 2883-2911, 2001.

[13] A. V. Aminova and N. A. M. Aminov, "Projective geometry of systems of differential equations: general conceptions," Tensor, vol. 62, no. 1, pp. 65-86, 2000.

[14] P. G. L. Leach, "A further note on the Hénon-Heiles problem," Journal of Mathematical Physics, vol. 22, no. 4, pp. 679-682, 1981.

[15] P. G. L. Leach and V. M. Gorringe, “The relationship between the symmetries of and the existence of conserved vectors for the equation $\ddot{x}+f(r) L+g(r) \widehat{r}=0$," Journal of Physics, vol. 23, no. 13, pp. 2765-2774, 1990.
[16] O. Gaponova and M. Nesterenko, "Systems of second-order ODEs invariant with respect to low-dimensional Lie algebras," Physics AUC, vol. 16, part 2, pp. 238-256, 2006.

[17] P. J. Olver, Applications of Lie Groups to Differential Equations, vol. 107 of Graduate Texts in Mathematics, Springer, New York, NY, USA, 2nd edition, 1993.

[18] V. Gorringe, V. M, and P. G. L. Leach, "Kepler's third law and the oscillator isochronism," American Journal of Physics, vol. 61, pp. 991-995, 1993.

[19] N. H. Ibragimov, F. M. Mahomed, D. P. Mason, and D. Sherwell, Di Erential Equations and Chaos, New Age International, New Delhi, India, 1996.

[20] K. S. Govinder and P. G. L. Leach, "Integrability of generalized Ermakov systems," Journal of Physics, vol. 27, no. 12, pp. 41534156, 1994. 


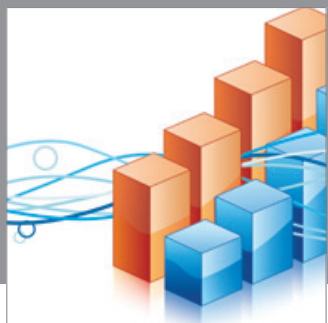

Advances in

Operations Research

mansans

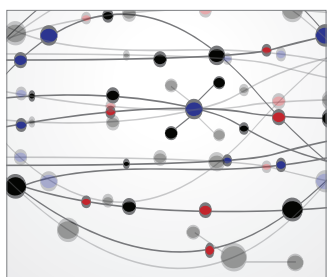

The Scientific World Journal
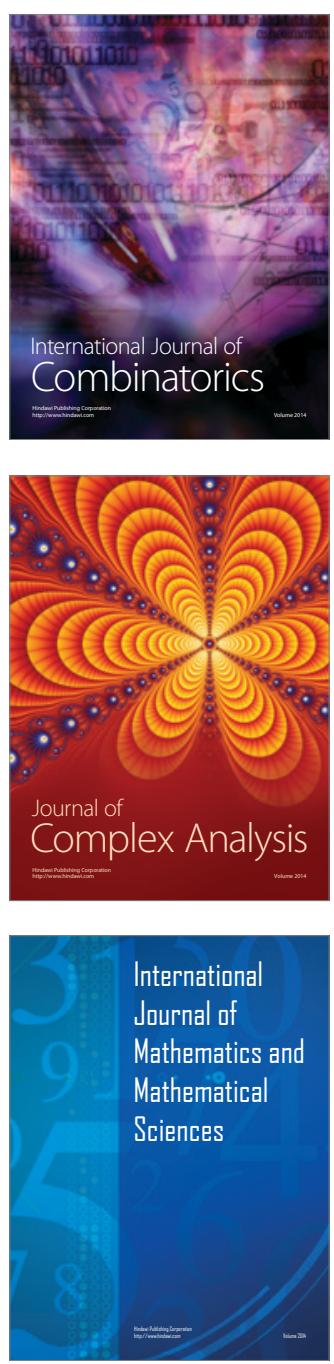
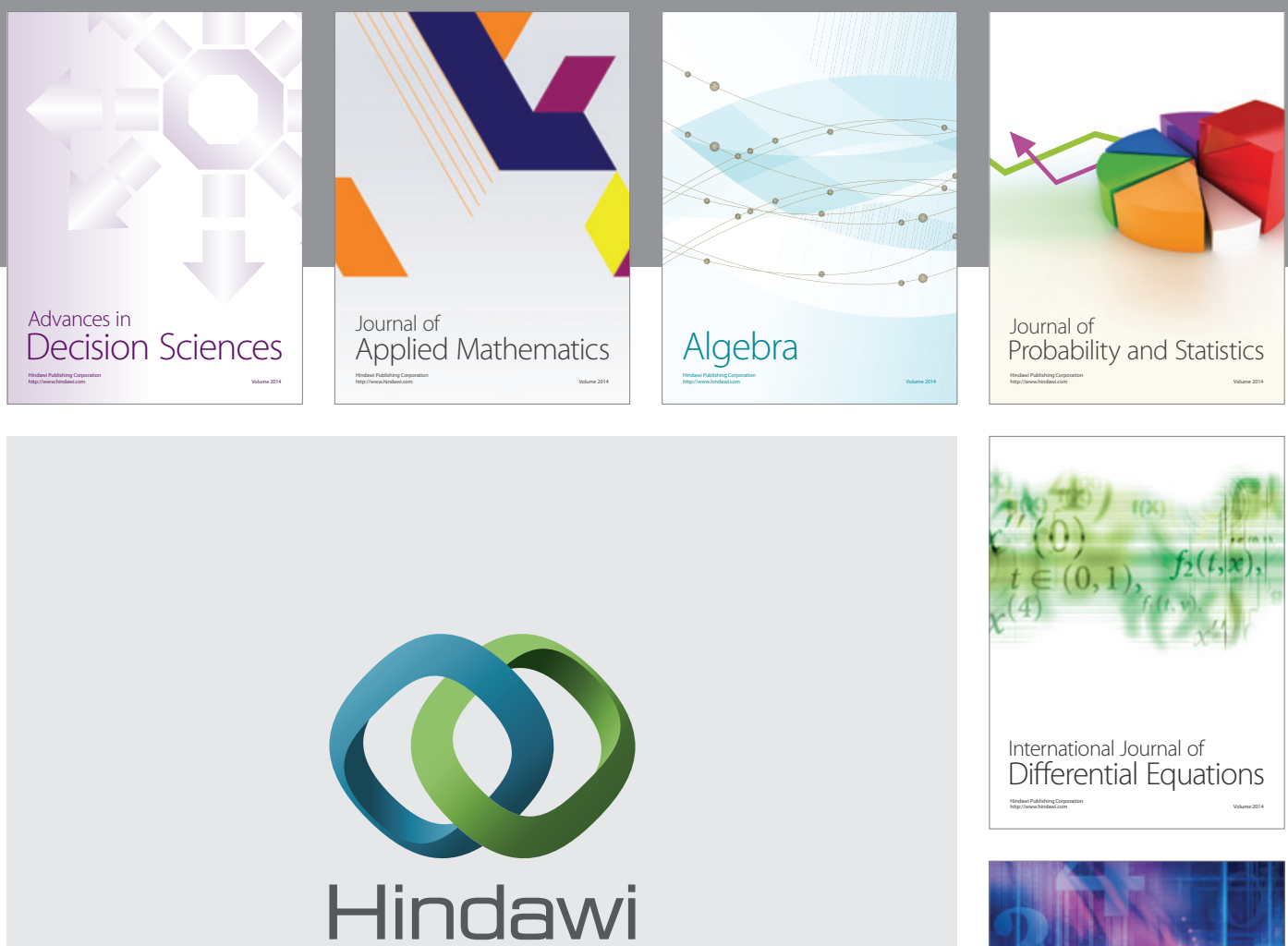

Submit your manuscripts at http://www.hindawi.com
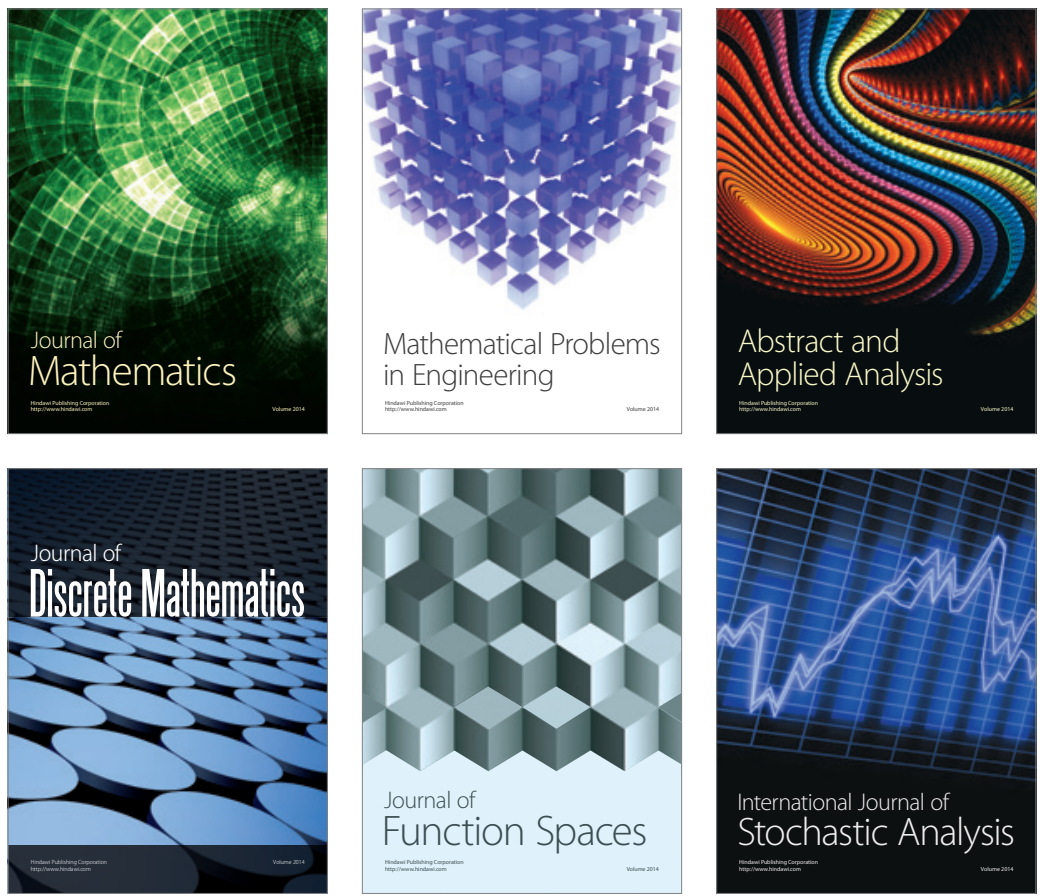

Journal of

Function Spaces

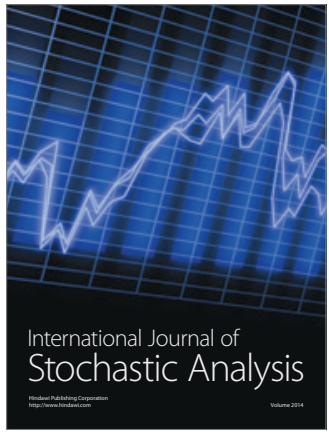

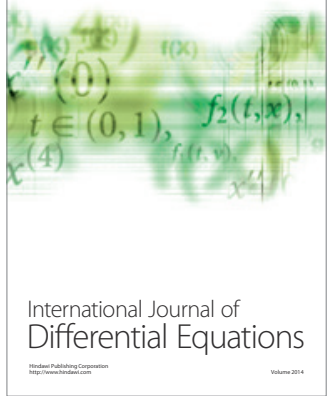
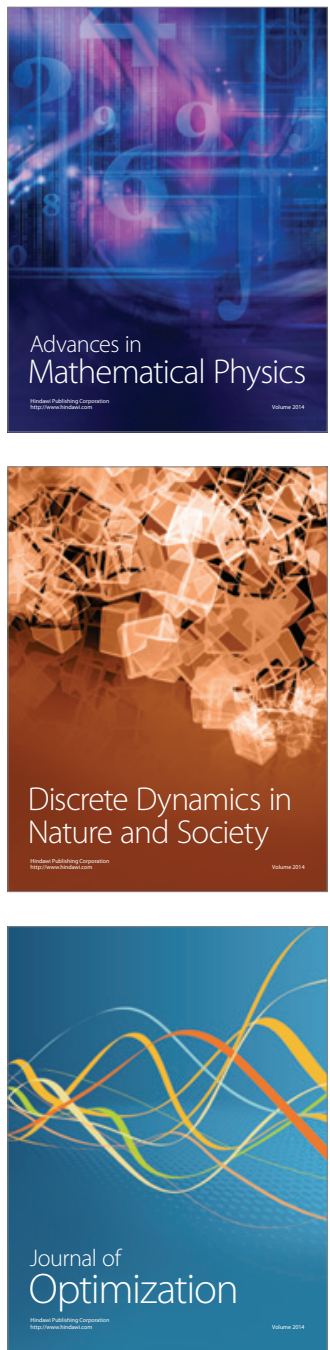